\title{
Probing doubly charged scalar bosons from the doublet at future high-energy colliders
}

\author{
Kazuki Enomoto®, ${ }^{*}$ Shinya Kanemura, ${ }^{\dagger}$ and Kento Katayama ${ }^{*}$ \\ Department of Physics, Osaka University, Toyonaka, Osaka 560-0043, Japan
}

(Received 26 February 2021; accepted 25 July 2021; published 31 August 2021)

\begin{abstract}
The isospin doublet scalar field with hypercharge $3 / 2$ is introduced in some new physics models such as tiny neutrino masses. Detecting the doubly charged scalar bosons from the doublet field can be a good probe of such models. However, their collider phenomenology has not been examined sufficiently. We investigate collider signatures of the doubly and singly charged scalar bosons at the LHC for the highluminosity upgraded option (HL-LHC) by looking at transverse mass distributions etc. With the appropriate kinematical cuts we demonstrate the background reduction in the minimal model in the following two cases depending on the mass of the scalar bosons. (1) The main decay mode of the singly charged scalar bosons is the tau lepton and missing (as well as charm and strange quarks). (2) That is into a top bottom pair. In the both cases, we assume that the doubly charged scalar boson is heavier than the singly charged ones. We conclude that the scalar doublet field with $Y=3 / 2$ is expected to be detectable at the HL-LHC unless the mass is too large.
\end{abstract}

DOI: 10.1103/PhysRevD.104.035040

\section{INTRODUCTION}

In spite of the success of the Standard Model (SM), there are good reasons to regard the model as an effective theory around the electroweak scale, above which the SM should be replaced by a model of new physics beyond the SM. Although a Higgs particle has been discovered at the LHC [1], the structure of the Higgs sector remains unknown. Indeed, the current data from the LHC can be explained in the SM. However, the Higgs sector in the SM causes the hierarchy problem, which must be solved by introducing new physics beyond the SM. In addition, the SM cannot explain gravity and several phenomena such as tiny neutrino masses, dark matter, baryon asymmetry of the universe, and so on. Clearly, extension of the SM is inevitable to explain these phenomena.

In the SM, introduction of a single isospin doublet scalar field is just a hypothesis without any theoretical principle. Therefore, there is still room to consider nonminimal shapes of the Higgs sector. When the above mentioned problems of the SM are considered together with such uncertainty of the Higgs sector, it might happen that it would be one of the

\footnotetext{
*kenomoto@ het.phys.sci.osaka-u.ac.jp

kanemu@het.phys.sci.osaka-u.ac.jp

k_katayama@het.phys.sci.osaka-u.ac.jp
}

Published by the American Physical Society under the terms of the Creative Commons Attribution 4.0 International license. Further distribution of this work must maintain attribution to the author(s) and the published article's title, journal citation, and DOI. Funded by SCOAP ${ }^{3}$. natural directions to think about the possibility of extended Higgs sectors as effective theories of unknown more fundamental theories beyond the SM. Therefore, there have been quite a few studies on models with extended Higgs sectors both theoretically and phenomenologically.

Additional isospin-multiplet scalar fields have often been introduced into the Higgs sector in lots of new physics models such as models of supersymmetric extensions of the $\mathrm{SM}$, those for tiny neutrino masses [2-12], dark matter [13-15], $C P$-violation [16,17], and the first-order phase transition $[18,19]$. One of the typical properties in such extended Higgs sector is a prediction of existence of charged scalar states. Therefore, theoretical study of these charged particles and their phenomenological exploration at experiments are essentially important to test these models of new physics.

There is a class of models with extended Higgs sectors in which doubly charged scalar states are predicted. They may be classified by the hypercharge of the isospin-multiplet scalar field in the Higgs sector; i.e., triplet fields with $Y=1$ $[3,4,8]$, doublet fields with $Y=3 / 2$ [20-25], and singlet fields with $Y=2[7,8,12,22]$. These fields mainly enter into new physics model motivated to explain tiny neutrino masses, sometimes together with dark matter and baryon asymmetry of the universe [12,20,21,23-25]. The doubly charged scalars are also introduced in models for other motivations [26,27]. Collider phenomenology of these models is important to discriminate the models. There have also been many studies along this line [20,28-37].

In this paper, we concentrate on the collider phenomenology of the model with an additional isodoublet field $\Phi$ 
with $Y=3 / 2$ at the high-luminosity-LHC (HL-LHC) with the collision energy of $\sqrt{s}=14 \mathrm{TeV}$ and the integrated luminosity of $\mathcal{L}=3000 \mathrm{fb}^{-1}$ [38]. Clearly, $\Phi$ cannot couple to fermions directly. The component fields are doubly charged scalar bosons $\Phi^{ \pm \pm}$and singly charged ones $\Phi^{ \pm}$. In order that the lightest one is able to decay into light fermions, we further introduce an additional doublet scalar field $\phi_{2}$ with the same hypercharge as of the SM one $\phi_{1}, Y=1 / 2$. Then, $Y=3 / 2$ component fields can decay via the mixing between two physical singly charged scalar states. Here, we define this model as a minimal model with doubly charged scalar bosons from the doublet. This minimal model has already been discussed in Ref. [20], where signal events via $p p \rightarrow W^{+*} \rightarrow \Phi^{++} H_{i}^{-}$have been analyzed, where $H_{i}^{ \pm}(i=1,2)$ are mass eigenstates of singly charged scalar states. They have indicated that masses of all the charged states $\Phi^{ \pm \pm}$and $H_{i}^{ \pm}$may be measurable from this single process by looking at the Jacobian peaks of transverse masses of several combinations of final states etc. However, they have not done any analysis for backgrounds. In this paper, we shall investigate both signal and backgrounds for this process to see whether or not the signal can dominate the backgrounds after performing kinematical cuts at the HL-LHC.

This paper is organized as follows. In Sec. II, we introduce the minimal model with doubly charged scalar bosons from the doublet which is mentioned above, and give a brief comment about current constraints on parameters in the scalar potential of the model from some experiments and theoretical issues. In Sec. III, we investigate decays of doubly and singly charged scalars and a production of doubly charged scalars at hadron colliders. In Sec. IV, results of numerical evaluations for the process $p p \rightarrow W^{+*} \rightarrow \Phi^{++} H_{i}^{-}$are shown. Final states of the process depend on mass spectrum of the charged scalars, and we investigate two scenarios with a benchmark value. Conclusions are given in Sec. V. In Appendix A, we show analytic formulas for decay rates of two-body and threebody decays of the charged scalars. In Appendix B, we discuss conditions for the scalar potential to be bounded from below. In Appendix C and D, constraints from the oblique parameters and the diphoton decay of the Higgs boson are investigated, respectively.

\section{MODEL OF THE SCALAR FIELD WITH $Y=3 / 2$}

We investigate the model whose scalar potential includes three isodoublet scalar fields $\phi_{1}, \phi_{2}$, and $\Phi$ [20]. Gauge groups and fermions in the model are same with those in the SM. Quantum numbers of scalar fields are shown in Table I. The hypercharge of two scalars $\phi_{1}$ and $\phi_{2}$ is $1 / 2$, and that of the other scalar $\Phi$ is $3 / 2$. In order to forbid the flavor changing neutral current (FCNC) at tree level, we impose the softly broken $Z_{2}$ symmetry, where $\phi_{2}$ and $\Phi$ have odd parity and $\phi_{1}$ has even parity [39].
TABLE I. The list of scalar fields in the model.

\begin{tabular}{lcccc}
\hline \hline & $S U(3)_{C}$ & $S U(2)_{L}$ & $U(1)_{Y}$ & $Z_{2}$ \\
\hline$\phi_{1}$ & $\mathbf{1}$ & $\mathbf{2}$ & $1 / 2$ & + \\
$\phi_{2}$ & $\mathbf{1}$ & $\mathbf{2}$ & $1 / 2$ & - \\
$\Phi$ & $\mathbf{1}$ & $\mathbf{2}$ & $3 / 2$ & - \\
\hline \hline
\end{tabular}

The scalar potential of the model is given by

$$
\begin{aligned}
V= & V_{\mathrm{THDM}}+\mu_{\Phi}^{2}|\Phi|^{2}+\frac{1}{2} \lambda_{\Phi}|\Phi|^{4} \\
& +\sum_{i=1}^{2} \rho_{i}\left|\phi_{i}\right|^{2}|\Phi|^{2}+\sum_{i=1}^{2} \sigma_{i}\left|\phi_{i}^{\dagger} \Phi\right|^{2} \\
& +\left\{\kappa\left(\Phi^{\dagger} \phi_{1}\right)\left(\tilde{\phi}_{1}^{\dagger} \phi_{2}\right)+\text { H.c. }\right\},
\end{aligned}
$$

where $V_{\text {THDM }}$ is the scalar potential in the two Higgs doublet model (THDM), and it is given by

$$
\begin{aligned}
V_{\mathrm{THDM}}= & \sum_{i=1}^{2} \mu_{i}^{2}\left|\phi_{i}\right|^{2}+\left(\mu_{3}^{2} \phi_{1}^{\dagger} \phi_{2}+\text { H.c. }\right) \\
& +\sum_{i=1}^{2} \frac{1}{2} \lambda_{i}\left|\phi_{i}\right|^{4}+\lambda_{3}\left|\phi_{1}\right|^{2}\left|\phi_{2}\right|^{2}+\lambda_{4}\left|\phi_{1}^{\dagger} \phi_{2}\right|^{2} \\
& +\frac{1}{2}\left\{\lambda_{5}\left(\phi_{1}^{\dagger} \phi_{2}\right)^{2}+\text { H.c. }\right\} .
\end{aligned}
$$

The $Z_{2}$ symmetry is softly broken by the terms of $\mu_{3}^{2} \phi_{1}^{\dagger} \phi_{2}$ and its Hermitian conjugate. Three coupling constants $\mu_{3}$, $\lambda_{5}$, and $\kappa$ can be complex number generally. After redefinition of phases of scalar fields, either $\mu_{3}$ or $\lambda_{5}$ remains as the physical $C P$-violating parameter. In this paper, we assume that this $C P$-violating phase is zero and all coupling constants are real for simplicity.

Component fields of the doublet fields are defined as follows;

$$
\phi_{i}=\left(\begin{array}{c}
\omega_{i}^{+} \\
\frac{1}{\sqrt{2}}\left(v_{i}+h_{i}+i z_{i}\right)
\end{array}\right), \quad \Phi=\left(\begin{array}{c}
\Phi^{++} \\
\Phi^{+}
\end{array}\right)
$$

where $i=1,2$. The fields $\phi_{1}$ and $\phi_{2}$ obtain the vacuum expectation values (VEVs) $v_{1} / \sqrt{2}$ and $v_{2} / \sqrt{2}$, respectively. These VEVs are described by $v \equiv \sqrt{v_{1}^{2}+v_{2}^{2}} \simeq$ $246 \mathrm{GeV}$ and $\tan \beta \equiv v_{2} / v_{1}$. On the other hand, the doublet $\Phi$ cannot have a VEV without violating electromagnetic charges spontaneously. ${ }^{1}$

\footnotetext{
${ }^{1}$ In this paper, we assume that the electric charge does not broken in the vacuum.
} 
From the stationary condition, we have

$$
\begin{aligned}
& \mu_{1}^{2}=-\mu_{3}^{2} \tan \beta+\frac{\lambda_{1}}{2} v^{2} c_{\beta}^{2}+\frac{\tilde{\lambda}}{2} v^{2} s_{\beta}^{2}, \\
& \mu_{2}^{2}=-\mu_{3}^{2} \cot \beta+\frac{\lambda_{2}}{2} v^{2} s_{\beta}^{2}+\frac{\tilde{\lambda}}{2} v^{2} c_{\beta}^{2},
\end{aligned}
$$

where

$$
\tilde{\lambda}=\lambda_{3}+\lambda_{4}+\lambda_{5}, \quad c_{\beta}=\cos \beta, \quad s_{\beta}=\sin \beta .
$$

Mass terms for the scalar fields are given by the followings.

$$
\begin{aligned}
V \ni & \frac{1}{2}\left(h_{1}, h_{2}\right) M_{h}^{2}\left(\begin{array}{l}
h_{1} \\
h_{2}
\end{array}\right)+\frac{1}{2}\left(z_{1}, z_{2}\right) M_{z}^{2}\left(\begin{array}{l}
z_{1} \\
z_{2}
\end{array}\right) \\
& +\left(\omega_{1}^{-}, \omega_{2}^{-}, \Phi^{-}\right) M_{\omega}^{2}\left(\begin{array}{c}
\omega_{1}^{+} \\
\omega_{2}^{+} \\
\Phi^{+}
\end{array}\right)+m_{\Phi}^{2}\left|\Phi^{++}\right|^{2},
\end{aligned}
$$

where $\tilde{\lambda}, c_{\beta}$, and $s_{\beta}$ are defined as

$$
\begin{gathered}
M_{h}^{2}=\left(\begin{array}{cc}
-\mu_{3}^{2} \tan \beta+\lambda_{1} v^{2} c_{\beta}^{2} & \mu_{3}^{2}+\tilde{\lambda} v^{2} c_{\beta} s_{\beta} \\
\mu_{3}^{2}+\tilde{\lambda} v^{2} c_{\beta} s_{\beta} & -\mu_{3}^{2} \cot \beta+\lambda_{2} v^{2} s_{\beta}^{2}
\end{array}\right), \\
M_{z}^{2}=\left(\mu_{3}^{2}+\lambda_{5} v^{2} c_{\beta} s_{\beta}\right)\left(\begin{array}{cc}
-\tan \beta & 1 \\
1 & -\cot \beta
\end{array}\right), \\
M_{\omega}=\left(\begin{array}{ccc}
-\mu_{\omega}^{2} \tan \beta & \mu_{\omega}^{2} & -\frac{\kappa}{2} v^{2} c_{\beta} s_{\beta} \\
\mu_{\omega}^{2} & -\mu_{\omega}^{2} \cot \beta & \frac{\kappa}{2} v^{2} c_{\beta}^{2} \\
-\frac{\kappa}{2} v^{2} c_{\beta} s_{\beta} & \frac{\kappa}{2} v^{2} c_{\beta}^{2} & m_{\Phi}^{2}
\end{array}\right), \\
m_{\Phi}^{2}=\mu_{\Phi}^{2}+\frac{v^{2}}{2}\left(\rho_{1} c_{\beta}^{2}+\rho_{2} s_{\beta}^{2}\right), \\
\mu_{\omega}^{2}=\mu_{3}^{2}+\frac{v^{2}}{2} c_{\beta} s_{\beta}\left(\lambda_{4}+\lambda_{5}\right) .
\end{gathered}
$$

The doubly charged scalar bosons $\Phi^{ \pm \pm}$are mass eigenstates without mixing, and their mass is given by $m_{\Phi}$. Mass eigenstates of the other scalars are defined by

$$
\begin{aligned}
&\left(\begin{array}{l}
H \\
h
\end{array}\right)=R(\alpha)\left(\begin{array}{l}
h_{1} \\
h_{2}
\end{array}\right), \quad\left(\begin{array}{l}
z \\
A
\end{array}\right)=R(\beta)\left(\begin{array}{l}
z_{1} \\
z_{2}
\end{array}\right), \\
&\left(\begin{array}{l}
\omega^{ \pm} \\
H_{1}^{ \pm} \\
H_{2}^{ \pm}
\end{array}\right)=\left(\begin{array}{ccc}
1 & 0 & 0 \\
0 & \cos \chi & \sin \chi \\
0 & -\sin \chi & \cos \chi
\end{array}\right) \\
& \times\left(\begin{array}{ccc}
\cos \beta & \sin \beta & 0 \\
-\sin \beta & \cos \beta & 0 \\
0 & 0 & 1
\end{array}\right)\left(\begin{array}{l}
\omega_{1}^{ \pm} \\
\omega_{2}^{ \pm} \\
\Phi^{ \pm}
\end{array}\right),
\end{aligned}
$$

where the left-hand sides of these equations are mass eigenstates, and the matrix $R(\theta)$ is the two-by-two rotation matrix for the angle $\theta$ :

$$
R(\theta)=\left(\begin{array}{cc}
\cos \theta & \sin \theta \\
-\sin \theta & \cos \theta
\end{array}\right)
$$

Mixing angles $\alpha$ and $\chi$ are defined as

$$
\begin{aligned}
& \tan 2 \alpha=\frac{2\left(M_{h}^{2}\right)_{12}}{\left(M_{h}^{2}\right)_{11}-\left(M_{h}^{2}\right)_{22}}, \\
& \tan 2 \chi=\frac{2\left(M_{\omega}^{2}\right)_{12}}{\left(M_{\omega}^{2}\right)_{11}-\left(M_{\omega}^{2}\right)_{22}} .
\end{aligned}
$$

In the following, for simplicity, we assume that $\sin (\beta-$ $\alpha)=1$ so that $h$ is the SM-like Higgs boson. Then, mass terms in Eq. (7) are diagonalized as

$$
\begin{aligned}
V \ni & \frac{1}{2} m_{h}^{2} h^{2}+\frac{1}{2} m_{H}^{2} H^{2}+\frac{1}{2} m_{A}^{2} A^{2}+m_{H_{1}}^{2}\left|H_{1}^{+}\right|^{2} \\
& +m_{H_{2}}^{2}\left|H_{2}^{+}\right|^{2}+m_{\Phi}^{2}\left|\Phi^{ \pm \pm}\right|^{2}
\end{aligned}
$$

where

$$
\begin{gathered}
m_{h}^{2}=v^{2}\left(\lambda_{1} c_{\beta}^{4}+2 \tilde{\lambda} s_{\beta}^{2} c_{\beta}^{2}+\lambda_{2} s_{\beta}^{4}\right), \\
m_{H}^{2}=m_{A}^{2}+v^{2}\left\{\left(\lambda_{1}+\lambda_{2}-2 \tilde{\lambda}\right) s_{\beta}^{2} c_{\beta}^{2}+\lambda_{5}\right\}, \\
m_{A}^{2}=-\frac{\mu_{3}^{2}}{s_{\beta} c_{\beta}}-\lambda_{5} v^{2}, \\
m_{H_{1}}^{2}=-\frac{\mu_{\omega}^{2}}{s_{\beta} c_{\beta}} \cos ^{2} \chi+\frac{1}{2} \kappa v^{2} c_{\beta} \sin 2 \chi+m_{\Phi}^{2} \sin ^{2} \chi, \\
m_{H_{2}}^{2}=-\frac{\mu_{\omega}^{2}}{s_{\beta} c_{\beta}} \sin ^{2} \chi-\frac{1}{2} \kappa v^{2} c_{\beta} \sin 2 \chi+m_{\Phi}^{2} \cos ^{2} \chi .
\end{gathered}
$$

The scalars $z$ and $\omega^{ \pm}$are Nambu-Goldstone bosons, and they are absorbed as the longitudinal components of $Z$ boson and $W^{ \pm}$bosons, respectively.

The doublet $\Phi$ does not have the Yukawa interaction with the SM fermions because of its hypercharge. ${ }^{2}$ Therefore, Yukawa interactions in the model is same with those in the THDM. They are divided into four types according to the $Z_{2}$ parities of each fermion (Type-I, II, X, and $Y$ [40]). In the following, we consider the Type-I Yukawa interaction where all left-handed fermions have even parity, and all right-handed ones have odd-parity. The type-I Yukawa interaction is given by

\footnotetext{
${ }^{2}$ If we consider higher dimensional operators, interactions between $\Phi$ and leptons are allowed [32].
} 


$$
\begin{aligned}
\mathcal{L}_{\text {Yukawa }}= & -\sum_{i, j=1}^{3}\left\{\left(Y_{u}\right)_{i j} \bar{Q}_{i L} \tilde{\phi}_{2} u_{j R}+\left(Y_{d}\right)_{i j} \overline{Q_{i L}} \phi_{2} d_{j R}\right. \\
& \left.+\left(Y_{\ell}\right)_{i j} \overline{L_{i L}} \phi_{2} \ell_{j R}\right\}+ \text { H.c. },
\end{aligned}
$$

where $Q_{i L}\left(L_{i L}\right)$ is the left-handed quark (lepton) doublet, and $u_{j R}, d_{j R}$, and $\ell_{j R}$ are the right-handed up-type quark, down-type quark and charged lepton fields, respectively. The Yukawa interaction of the singly charged scalars are given by

$$
\begin{aligned}
& -\frac{\sqrt{2}}{v} \cot \beta \sum_{i, j=1}^{3}\left\{V_{u_{i} d_{j}} \overline{u_{i}}\left(m_{u_{i}} P_{L}+m_{d_{j}} P_{R}\right) d_{j}\right. \\
& \left.\quad+\delta_{i j} m_{\ell_{i}} \overline{\nu_{i}} P_{L} \ell_{i}\right\}\left(\cos \chi H_{1}^{+}-\sin \chi H_{2}^{+}\right)+\text {H.c. },
\end{aligned}
$$

where $V_{u_{i} d_{j}}$ is the $\left(u_{i}, d_{j}\right)$ element of the CabibboKobayashi-Maskawa $(\mathrm{CKM})$ matrix $[16,41], \delta_{i j}$ is the Kroneker delta, and $P_{L}\left(P_{R}\right)$ is the chirality projection operator for left-handed (right-handed) chirality. In addition, $\left(u_{1}, u_{2}, u_{3}\right)=(u, c, t)$ are the up-type quarks, $\left(d_{1}, d_{2}, d_{3}\right)=(d, s, b)$ are the down-type quarks, $\left(\ell_{1}, \ell_{2}, \ell_{3}\right)=(e, \mu, \tau)$ are the charged leptons, and $\left(\nu_{1}, \nu_{2}, \nu_{3}\right)=\left(\nu_{e}, \nu_{\mu}, \nu_{\tau}\right)$ are the neutrinos. The symbols $m_{u_{i}}, m_{d_{i}}$, and $m_{\ell_{i}}$ are the masses for $u_{i}, d_{i}$, and $\ell_{i}$, respectively. In the following discussions, we neglect nondiagonal terms of the CKM matrix.

Finally, we discuss theoretical and experimental constrains on some parameters in the model. If the coupling constant $\kappa$ in the scalar potential is zero, the model have a new discrete $Z_{2}$ symmetry where the doublet $\Phi$ is odd and all other fields are even. This $Z_{2}$ symmetry stabilizes $\Phi^{ \pm \pm}$ or $\Phi^{ \pm}$, and their masses and interactions are strongly constrained. Thus, $\kappa \neq 0$ is preferred, and it means that $\sin \chi \neq 0$. In this paper, we assume that $\chi=\pi / 4$ just for simplicity. Some quartic scalar couplings are determined by the masses of new scalars by using the mass formulas in the above. In addition, quartic couplings have to satisfy conditions for the potential to be bounded from below. In Appendix B, these conditions are discussed in detail. The masses of $H_{1,2}^{ \pm}$are strongly constrained by flavor experiments in the region where $\tan \beta \lesssim 2$ [42-44]. In larger $\tan \beta$ regions, the constraints for $m_{H_{1,2}}$ are given by direct searches of $H_{1,2}^{ \pm}$at high energy colliders [44,45]. From the LEP, the lower limit for $m_{H_{1,2}}$ is given by about $80 \mathrm{GeV}$ [46]. The direct search at the LHC give the upper limit for $\sigma_{H^{ \pm}} \times$(Branching ratio), where $\sigma_{H^{ \pm}}$is the cross section of a production process for $H_{1,2}^{ \pm}$. The upper limit for the decay channel $H_{1,2}^{ \pm} \rightarrow \tau \nu\left(H_{1,2}^{ \pm} \rightarrow t b\right)$ is given by Ref. [47] (Ref. [48]), and at 95\% C.L., it is about $3.0 \mathrm{pb}$ (3.6 pb) for $m_{H_{1.2}}=100 \mathrm{GeV}$ (200 GeV). Furthermore, the new scalars can affect some observables at loop levels. For example, they contribute to the oblique parameters

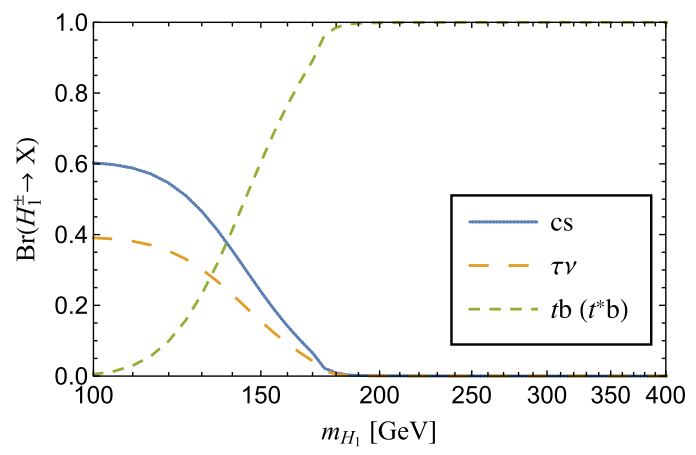

FIG. 1. The branching ratio of $H_{1}^{ \pm}$.

$(S, T$, and $U$ ) [49] and the diphoton decay of the Higgs boson. Some parameters in the scalar potential are constrained by measurements of these observables. We discuss details of these constraints in Appendix C and D.

\section{PRODUCTION AND DECAYS OF CHARGED SCALAR STATES}

In this section, we investigate the decay of the new charged scalars and the production of the doubly charged scalar at hadron colliders. In the following discussion, we assume that $\Phi^{ \pm \pm}$is heavier than $H_{1}^{ \pm}$and $H_{2}^{ \pm}$. Then, $H_{1,2}^{ \pm}$ cannot decay into $\Phi^{ \pm \pm}$. In addition, we assume that $H$ and $A$ are so heavy that they do not affect significantly the decay modes of all the charged scalars.

\section{A. Decays of charged scalar sates}

First, we discuss the decays of the singly charged scalars $H_{1}^{ \pm}$and $H_{2}^{ \pm}$. They decay into the SM fermions via Yukawa interaction in Eq. (25). Since they are lighter than $\Phi^{ \pm \pm}, H$, and $A$, their decays into $\Phi^{ \pm \pm} W^{\mp(*)}, H W^{ \pm(*)}$, and $A W^{ \pm(*)}$ are prohibited. On the other hand, the decay of the heavier singly charged scalars into the lighter one and $Z^{(*)}$ is allowed, and it is generated via the gauge interaction. In the following, we assume that $H_{2}^{ \pm}$is heavier than $H_{1}^{ \pm}\left(m_{H_{2}}>m_{H_{1}}\right)$.

In Fig. 1, the branching ratio for each decay channel of $H_{1}^{ \pm}$is shown. Since we assume that $H_{1}^{ \pm}$is lighter than $H_{2}^{ \pm}$, it decays via the Yukawa interaction [40]. ${ }^{3}$ In the region where $m_{H_{1}} \lesssim 140 \mathrm{GeV}$, the decay into $c s$ and that into $\tau \nu$ are dominant. When we consider a little heavier $H_{1}^{ \pm}$, which are in the mass region between $140 \mathrm{GeV}$ and $m_{t}+m_{b} \simeq 180 \mathrm{GeV}$, the branching ratio for $H_{1,2}^{ \pm} \rightarrow t^{*} b \rightarrow W^{ \pm} b \bar{b}$ is dominant [51]. ${ }^{4}$ In the mass

\footnotetext{
${ }^{3}$ In this paper, we neglect the effects of one-loop induced decays $H_{i}^{ \pm} \rightarrow W^{ \pm} \gamma$ and $H_{i} \pm \rightarrow W^{ \pm} Z$ [50].

${ }^{4}$ In Ref [51], type-II Yukawa interaction is investigated, and the condition $\tan \beta \lesssim 1$ is needed to make the decay $H_{1,2}^{ \pm} \rightarrow t^{*} b$ dominant. In our case (type-I), this condition is not necessary because all fermions couple to $\phi_{2}$ universally.
} 

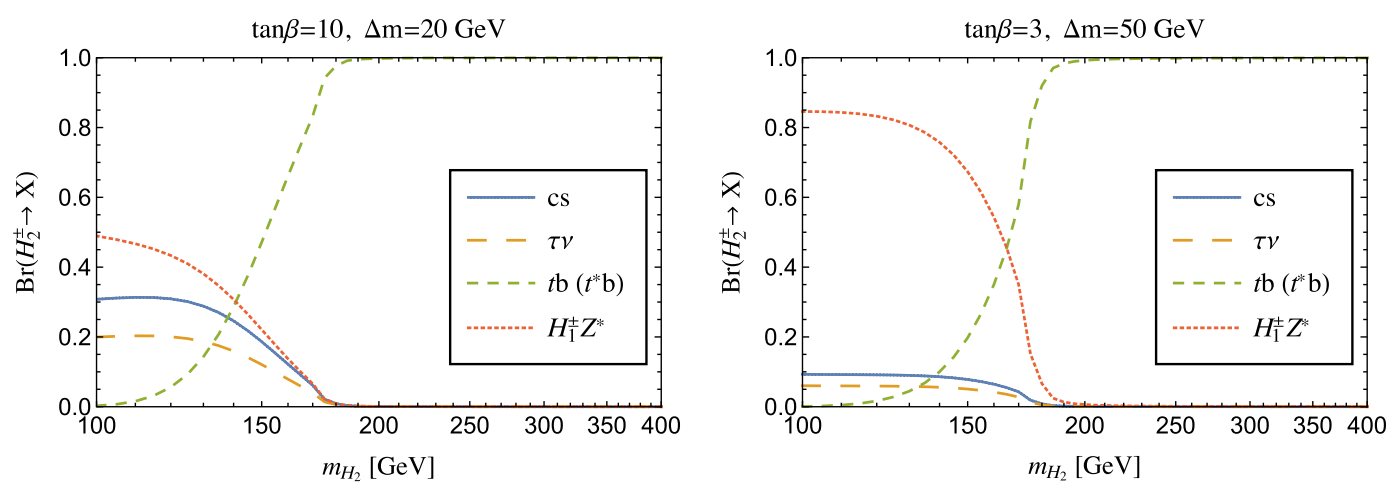

FIG. 2. The branching ratio of $H_{2}^{ \pm}$. In the left figure, we assume that $\Delta m\left(\equiv m_{H_{2}}-m_{H_{1}}\right)=20 \mathrm{GeV}$ and $\tan \beta=10$. In the right figure, we assume that $\Delta m=50 \mathrm{GeV}$ and $\tan \beta=3$

region $m_{t}+m_{b}<m_{H_{1}}$, the branching ratio for $H_{1}^{ \pm} \rightarrow t b$ is almost $100 \%$. The decays into $c s, \tau \nu$, and $t^{(*)} b$ are all induced by the Yukawa interaction. Since we consider the type-I Yukawa interaction, the dependence on $\tan \beta$ of each decay channel is same. Thus, the branching ratio in Fig. 1 hardly depends on the value of $\tan \beta$. Analytic formulas of decay rates for each decay channel are shown in Appendix A 1.

The singly charged scalar $H_{2}^{ \pm}$also decays into the SM fermions via the Yukawa interaction. In addition, $H_{2}^{ \pm} \rightarrow$ $H_{1}^{ \pm} Z^{(*)}$ is allowed. In Fig. 2, the branching ratios of $H_{2}^{ \pm}$in two cases are shown. The left figure of Fig. 2 is for $\tan \beta=$ 10 and $\Delta m\left(\equiv m_{H_{2}}-m_{H_{1}}\right)=20 \mathrm{GeV}$. In the small mass region, the decay $H_{2}^{ \pm} \rightarrow H_{1}^{ \pm} Z^{*}$ is dominant. In the region where $m_{H_{2}} \gtrsim 140 \mathrm{GeV}$, the decay $H_{2}^{ \pm} \rightarrow t^{(*)} b$ becomes dominant, and the branching ratio for $H_{2}^{ \pm} \rightarrow t b$ is almost $100 \%$ for $m_{H_{2}} \gtrsim 180 \mathrm{GeV}$. If we consider smaller $\tan \beta$, the decays via Yukawa interaction are enhanced because the Yukawa interaction is proportional to $\cot \beta$. [See Eq. (25).] Thus, he branching ratio for $H_{2}^{ \pm} \rightarrow H_{1}^{ \pm} Z^{*}$ decreases.

The right figure of Fig. 2 is for the case where $\tan \beta=3$ and $\Delta m=50 \mathrm{GeV}$. In the small mass region, the branching ratio for $H_{2}^{ \pm} \rightarrow H_{1}^{ \pm} Z^{*}$ is about $80 \%$, and those for other decay channels are negligibly small. However, in the mass region where $m_{H_{2}} \gtrsim 180 \mathrm{GeV}, H_{2}^{ \pm} \rightarrow H_{1}^{ \pm} Z^{*}$ become negligibly small, and the branching ratio for $H_{2}^{ \pm} \rightarrow t b$ is almost $100 \%$. If we consider larger $\tan \beta$, the decays via the Yukawa interaction is suppressed, and the branching ratio for $\mathrm{H}_{2}^{ \pm} \rightarrow$ $H_{1}^{ \pm} Z^{*}$ increases. Thus, the crossing point of the branching ratio for $H_{2}^{ \pm} \rightarrow t b\left(t^{*} b\right)$ and that for $H_{2}^{ \pm} \rightarrow H_{1}^{ \pm} Z^{*}$ move to the point at heavier $m_{\mathrm{H}_{2}}$. Analytic formulas of decay rates for each decay channel are shown in Appendix A 1.

Next, we discuss the decay of the doubly charged scalar $\Phi^{ \pm \pm}$. The doubly charged scalar $\Phi^{ \pm \pm}$does not couple to fermions via Yukawa interaction. ${ }^{5}$ Therefore, it decays via

\footnotetext{
${ }^{5}$ This is different from doubly charged Higgs boson in the triplet model in which dilepton decays of doubly charged Higgs bosons are important signature to test the model [36].
}

the weak gauge interaction. ${ }^{6}$ We consider the following three cases.

First, the case where $\Delta m_{1}\left(\equiv m_{\Phi}-m_{H_{1}}\right)<80 \mathrm{GeV}$ and $\Delta m_{2}\left(\equiv m_{\Phi}-m_{H_{2}}\right)<80 \mathrm{GeV}$ is considered. In this case, $\Phi^{ \pm \pm}$cannot decay into the on-shell $H_{1,2}^{ \pm}$, and three-body decays are dominant. In the upper left figure of Fig. 3, the branching ratio of $\Phi^{ \pm \pm}$in this case is shown. We assume that $\tan \beta=3, \Delta m_{1}<20 \mathrm{GeV}, \Delta m_{2}<10 \mathrm{GeV}$. In the small mass region, $\Phi^{ \pm \pm} \rightarrow H_{1}^{ \pm} f f$ is dominant. With increasing of $m_{\Phi}$, the masses of $H_{1,2}^{ \pm}$also increase because the mass differences between them are fixed. Thus, the branching ratio for $\Phi^{ \pm \pm} \rightarrow W^{ \pm} f f$ is dominant in the large mass region. At the point $m_{\Phi} \simeq 260 \mathrm{GeV}$, the branching ratio for $\Phi^{ \pm \pm} \rightarrow W^{ \pm} f f$ changes rapidly. It is because that at this point, the decay channel $\Phi^{ \pm \pm} \rightarrow W^{ \pm} t b$ is open. If we consider the large $\tan \beta$, the decay rates of $\Phi^{ \pm \pm} \rightarrow$ $W^{\mp} f f$ becomes small because this process includes $H_{1,2}^{ \pm *} \rightarrow f f$ via Yukawa interaction which is proportional to $\cot \beta$. However, the decays $\Phi^{ \pm \pm} \rightarrow H_{1,2}^{ \pm} f f$ are generated via only the gauge interaction. Thus, for $\tan \beta \gtrsim 3$, the branching ratio for $\Phi^{ \pm \pm} \rightarrow W^{ \pm} f f$ becomes small.

Second, the case where $\Delta m_{1}>80 \mathrm{GeV}$ and $\Delta m_{2}<$ $80 \mathrm{GeV}$ is considered. In this case, $\Phi^{ \pm \pm} \rightarrow H_{1}^{ \pm} W^{ \pm}$is allowed while $\Phi^{ \pm \pm} \rightarrow H_{2}^{ \pm} W^{ \pm}$is prohibited. In the upper right figure of Fig. 3, the branching ratio of $\Phi^{ \pm \pm}$in this case is shown. We assume that $\tan \beta=3, \Delta m_{1}<100 \mathrm{GeV}$, $\Delta m_{2}<50 \mathrm{GeV}$. In all mass region displayed in the figure, the branching ratio for $\Phi^{ \pm \pm} \rightarrow H_{1}^{ \pm} W^{ \pm}$are almost $100 \%$, and those for other channels are at most about $0.1 \%$. At the point $m_{\Phi} \simeq 260 \mathrm{GeV}$, the branching ratio for $\Phi^{ \pm \pm} \rightarrow$ $W^{ \pm} f f$ changes rapidly. It is because that at this point, the decay channel $\Phi^{ \pm \pm} \rightarrow W^{ \pm} t b$ is open.

Third, the case where $\Delta m_{1}>80 \mathrm{GeV}$ and $\Delta m_{2}>$ $80 \mathrm{GeV}$ is considered. and both of $\Phi^{ \pm \pm} \rightarrow H_{1,2}^{ \pm} W^{ \pm}$are

\footnotetext{
${ }^{6}$ In triplet Higgs models, if the VEV of the triplet field is small enough the main decay mode of the doubly charged Higgs boson is the diboson decay [31]. On the other hand, in our model, such a decay mode does not exist at tree level.
} 

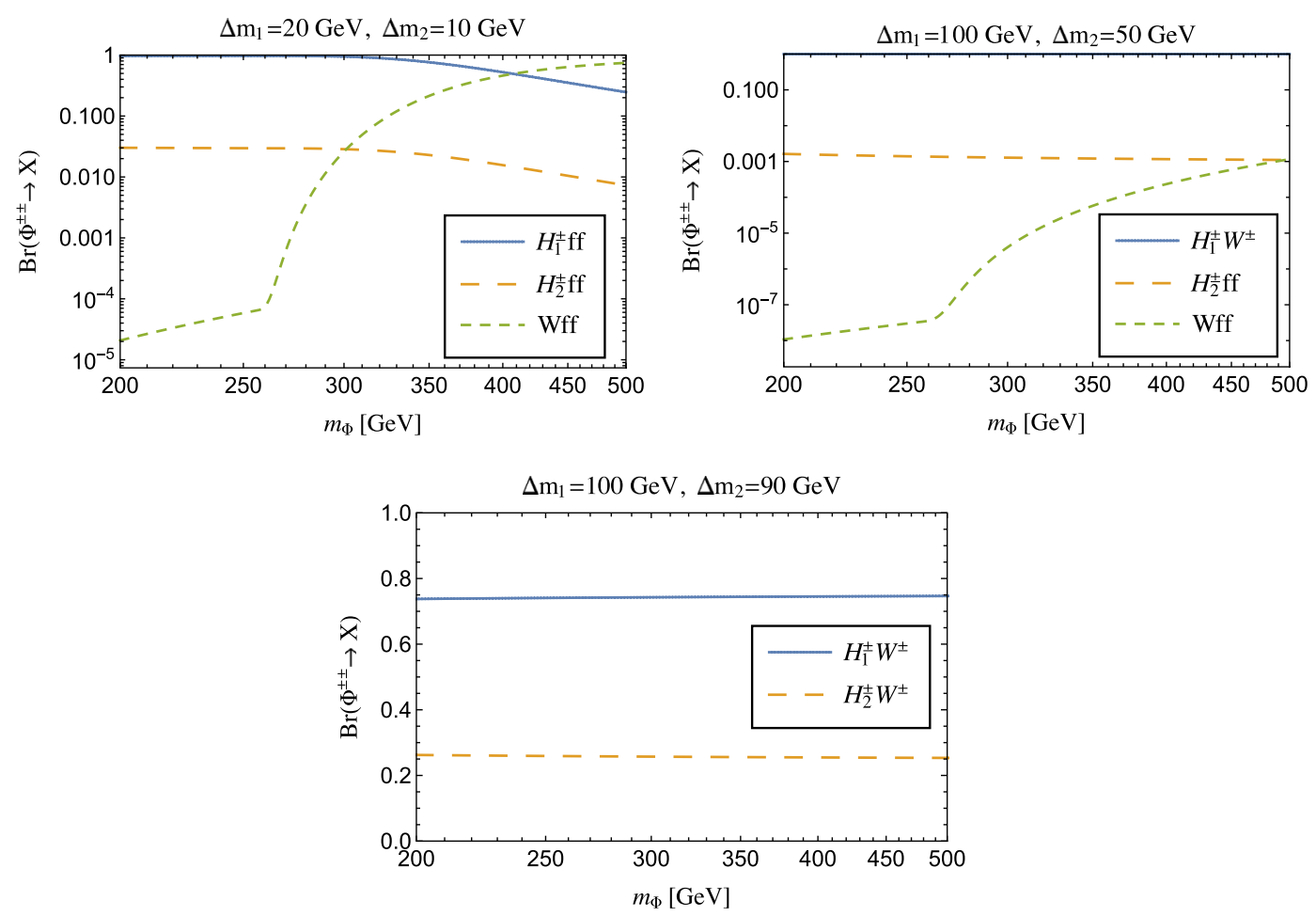

FIG. 3. The branching ratios of the decay of $\Phi^{ \pm \pm}$. The upper left (right) figure is those in the case that $\Delta m_{1}\left(\equiv m_{\Phi}-m_{H_{1}}\right)=20 \mathrm{GeV}$ $(100 \mathrm{GeV})$ and $\Delta m_{2}\left(\equiv m_{\Phi}-m_{H_{2}}\right)=10 \mathrm{GeV} \quad(50 \mathrm{GeV})$. The bottom one corresponds to the case that $\Delta m_{1}=100 \mathrm{GeV}$ and $\Delta m_{2}=90 \mathrm{GeV}$.

allowed. In the lower figure of Fig. 3, the branching ratio in this case is shown. We assume that $\tan \beta=3, \Delta m_{1}=$ $100 \mathrm{GeV}, \Delta m_{2}=90 \mathrm{GeV}$. In all mass region displayed in the figure, the branching ratio does not change because the mass differences between $\Phi^{ \pm \pm}$and $H_{1,2}^{ \pm}$are fixed. The branching ratio for $\Phi^{ \pm \pm} \rightarrow H_{1}^{ \pm} W^{ \pm}$is about $75 \%$, and that for $\Phi^{ \pm \pm} \rightarrow H_{2}^{ \pm} W^{ \pm}$is about $25 \%$. These decays are generated via only the gauge interaction. Thus, the branching ratios of them do not depend on $\tan \beta$, and they are determined by only the mass differences between $\Phi^{ \pm \pm}$and $m_{H_{1,2}}$.

\section{B. Production of $\boldsymbol{\Phi}^{ \pm \pm}$at hadron colliders}

We here discuss the production of the doubly charged scalar $\Phi^{ \pm \pm}$. In our model, production processes of charged scalar states are $p p \rightarrow W^{+*} \rightarrow H_{i}^{+} A(H), p p \rightarrow Z^{*}(\gamma) \rightarrow$ $H_{i}^{+} H_{i}^{-}, \quad p p \rightarrow W^{+*} \rightarrow \Phi^{++} H_{i}^{-}, \quad$ and $\quad p p \rightarrow Z^{*}(\gamma) \rightarrow$ $\Phi^{++} \Phi^{--}$. In the THDM, the first and second processes (the singly charged scalar production) can also occur $[52,53]$ However, doubly charged scalar bosons are not included in the THDM. ${ }^{7}$ In the model with the isospin

\footnotetext{
${ }^{7}$ In the THDM, and also in our model with the $Y=3 / 2$ doublet, there are also single production processes of singly charged Higgs bosons such as $g b \rightarrow t H^{ \pm}$[54], $q b \rightarrow q^{\prime} b H^{ \pm}$ [55], $b \bar{b} \rightarrow W^{ \pm} H^{\mp}$ [56,57], $g g \rightarrow W^{ \pm} H^{\mp}$ [57,58], etc. (See also Ref. [59].) In this paper, we do not consider these processes and concentrate only on the processes $p p \rightarrow W^{+*} \rightarrow \Phi^{++} H_{i}^{-}$.
}

triplet scalar with $Y=1[3,4,8,26,27]$, all of these production processes can appear. However, the main decay mode of doubly charged scalar is different from our model. In the triplet model, the doubly charged scalar from the triplet mainly decays into dilepton [36] or diboson [31]. In our model, on the other hand, $\Phi^{ \pm \pm}$mainly decays into the singly charged scalar and $W$ boson.

In this paper, we investigate the associated production $p p \rightarrow W^{+*} \rightarrow \Phi^{++} H_{i}^{-}(i=1,2)$. In this process, information on masses of all the charged states $\Phi^{ \pm \pm}$and $H_{i}^{ \pm}$ appear in the Jacobian peaks of transverse masses of several combinations of final states [20]. Pair productions are also important in searching for $\Phi^{ \pm \pm}$and $H_{i}^{ \pm}$, however we focus on the associated production in this paper. The parton-level cross section of the process $q \bar{q}^{\prime} \rightarrow W^{+*} \rightarrow \Phi^{++} H_{i}^{-}(i=1,2)$ is given by

$$
\begin{aligned}
\sigma_{i}= & \frac{G_{F}^{2} m_{W}^{4}\left|V_{q q^{\prime}}\right|^{2} \chi_{i}^{2}}{12 \pi s^{2}\left(s-m_{W}^{2}\right)^{2}} \\
& \times\left[m_{H_{i}^{ \pm}}^{4}+\left(s-m_{\Phi^{ \pm \pm}}^{2}\right)^{2}-2 m_{H_{i}^{ \pm}}^{2}\left(s+m_{\Phi^{ \pm \pm}}^{2}\right)\right]^{3 / 2},
\end{aligned}
$$

where $s$ is the square of the center-of-mass energy, $G_{F}$ is the Fermi coupling constant, and $V_{q q^{\prime}}$ is the $\left(q, q^{\prime}\right)$ element of CKM matrix. In addition, $\chi_{i}$ in Eq. (26) is defined as

$$
\chi_{1}=\sin \chi, \quad \chi_{2}=\cos \chi
$$




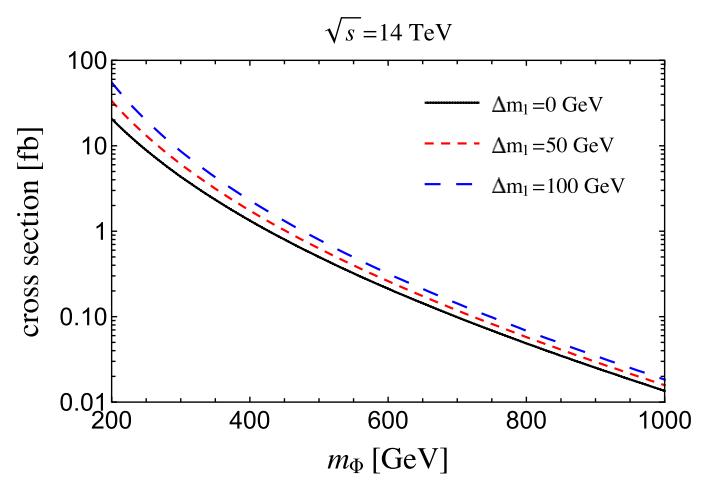

FIG. 4. The cross section for $p p \rightarrow W^{+*} \rightarrow \Phi^{++} H_{1}^{-}$, where $\sqrt{s}=14 \mathrm{TeV}$ and $\chi=\pi / 4$. The black, red, blue lines are those in the case that $\Delta m_{1}\left(\equiv m_{\Phi}-m_{H_{1}}\right)=0,50$, and $100 \mathrm{GeV}$, respectively.

In Fig. 4, we show the cross section for $p p \rightarrow W^{+*} \rightarrow$ $\Phi^{++} H_{1}^{-}$in the case that $\sqrt{s}=14 \mathrm{TeV}$ and $\chi=\pi / 4$. The cross section is calculated by using MadGraph5_aMC@NLO [60] and FEYNRULES [61]. The black, red, blue lines are those in the case that $\Delta m_{1}=0,50$, and $100 \mathrm{GeV}$, respectively. The results in Fig. 4 do not depend on the value of $\tan \beta$. At the HL-LHC $\left(\sqrt{s}=14 \mathrm{TeV}\right.$ and $\left.\mathcal{L}=3000 \mathrm{fb}^{-1}\right)$, about the $6 \times 10^{4}$ doubly charged scalars are expected to be generated in the case that $m_{\Phi}=200 \mathrm{GeV}$ and $\Delta m_{1}=50 \mathrm{GeV}$. If $\Phi^{ \pm \pm}$is heavier, the cross section decreases, and about the 300 doubly charged scalars are expected to be generated at the HL-LHC in the case that $m_{\Phi}=800 \mathrm{GeV}$. The cross section increases with increasing of the mass difference $\Delta m_{1}$. Since we assume that $\chi=\pi / 4$, the cross section of the process $p p \rightarrow W^{+*} \rightarrow \Phi^{++} H_{2}^{-}$is same with that in Fig. 4 if $m_{H_{2}}=m_{H_{1}}$. If we consider the case that $|\sin \chi|>|\cos \chi|(|\cos \chi|>|\sin \chi|)$, the cross section of $p p \rightarrow W^{+*} \rightarrow \Phi^{++} H_{1}^{-}$become larger (smaller) than that of $p p \rightarrow W^{+*} \rightarrow \Phi^{++} H_{2}^{-}$even if $m_{H_{2}}=m_{H_{1}}$.

\section{SIGNAL AND BACKGROUNDS AT HL-LHC}

In this section, we investigate the detectability of the process $p p \rightarrow W^{+*} \rightarrow \Phi^{++} H_{i}^{-}(i=1,2)$ in two benchmark scenarios. In the first scenario (Scenario I), the masses of $H_{1}^{ \pm}$and $H_{2}^{ \pm}$are set to be $100 \mathrm{GeV}$ and $120 \mathrm{GeV}$, so that they cannot decay into $t b$. In this case, their masses are so small that the branching ratio for three body decay $H_{1,2}^{ \pm} \rightarrow$ $W^{ \pm} b \bar{b}$ is less than $5 \%$ approximately. Thus, their main decay modes are $H_{1,2}^{ \pm} \rightarrow c s$ and $H_{1,2}^{ \pm} \rightarrow \tau \nu$. In the second scenario (Scenario II), masses of $H_{1}^{ \pm}$and $H_{2}^{ \pm}$are set to be $200 \mathrm{GeV}$ and $250 \mathrm{GeV}$, and they predominantly decay into $t b$ with the branching ratio to be almost $100 \%$.

In our analysis below, we assume the collider performance at HL-LHC as follows [38];

$$
\sqrt{s}=14 \mathrm{TeV}, \quad \mathcal{L}=3000 \mathrm{fb}^{-1},
$$

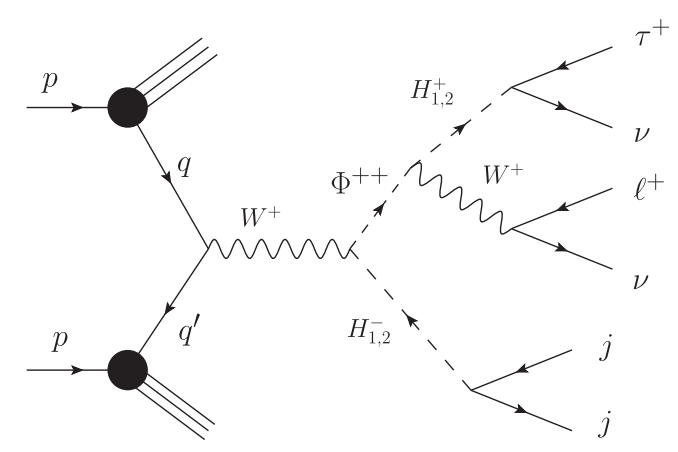

FIG. 5. The Feynman diagram for the signal process in Scenario I, where $q$ and $q^{\prime}$ are partons.

where $\sqrt{s}$ is the center-of-mass energy and $\mathcal{L}$ is the integrated luminosity. Furthermore, we use the following kinematical cuts (basic cuts) for the signal event [60];

$$
\begin{aligned}
p_{T}^{j} & >20 \mathrm{GeV}, \quad p_{T}^{\ell}>10 \mathrm{GeV}, \quad\left|\eta_{j}\right|<5, \quad\left|\eta_{\ell}\right|<2.5 \\
\Delta R_{j j} & >0.4, \quad \Delta R_{\ell j}>0.4, \quad \Delta R_{\ell \ell}>0.4,
\end{aligned}
$$

where $p_{T}^{j}\left(p_{T}^{\ell}\right)$ and $\eta_{j}\left(\eta_{\ell}\right)$ are the transverse momentum and the pseudorapidity of jets (charged leptons), respectively, and $\Delta R_{j j}, \Delta R_{\ell j}$, and $\Delta R_{\ell \ell}$ in Eq. (29) are the angular distances between two jets, charged leptons and jets, and two charged leptons, respectively.

\section{A. Scenario I}

In this scenario, the singly charged scalars decay into $c s$ or $\tau \nu$ dominantly. (See Figs. 1 and 2.) We investigate the process $p p \rightarrow W^{+*} \rightarrow \Phi^{++} H_{1,2}^{-} \rightarrow$ $\tau^{+} \ell^{+} \nu \nu j j(\ell=e, \mu)$. The Feynman diagram for the process is shown in Fig. 5. In this process, the doubly charged scalar $\Phi^{++}$and one of the singly charged scalars $H_{1,2}^{-}$are generated via s-channel $W^{+*}$. The produced singly charged scalar decays into a pair of jets, and $\Phi^{++}$ decays into $\tau^{+} \ell^{+} \nu \nu$ through the on-shell pair of the singly charged scalar and $W^{+}$. Thus, in the distribution of the transverse mass of $\tau^{+} \ell^{+} \mathscr{E}_{T}$, where $E_{T}$ is the missing transverse energy, we can see the Jacobian peak whose endpoint corresponds to $m_{\Phi}[20] .{ }^{8}$ In the present process, furthermore, in the distribution of the transverse mass of

\footnotetext{
${ }^{8}$ In general, the transverse mass $M_{T}$ of $n$ particles is defined as follows;

$$
\begin{gathered}
M_{T}^{2}=\left(E_{T 1}+E_{T 2}+\cdots+E_{T n}\right)^{2}+\left|\boldsymbol{p}_{T 1}+\boldsymbol{p}_{T 2}+\cdots+\boldsymbol{p}_{T n}\right|^{2}, \\
E_{T i}^{2}=\left|\boldsymbol{p}_{T i}\right|^{2}+m_{i}^{2}(i=1,2, \ldots, n),
\end{gathered}
$$

where $\boldsymbol{p}_{T i}$ and $m_{i}$ are the transverse momentum and the mass of $i$ th particle, respectively. 

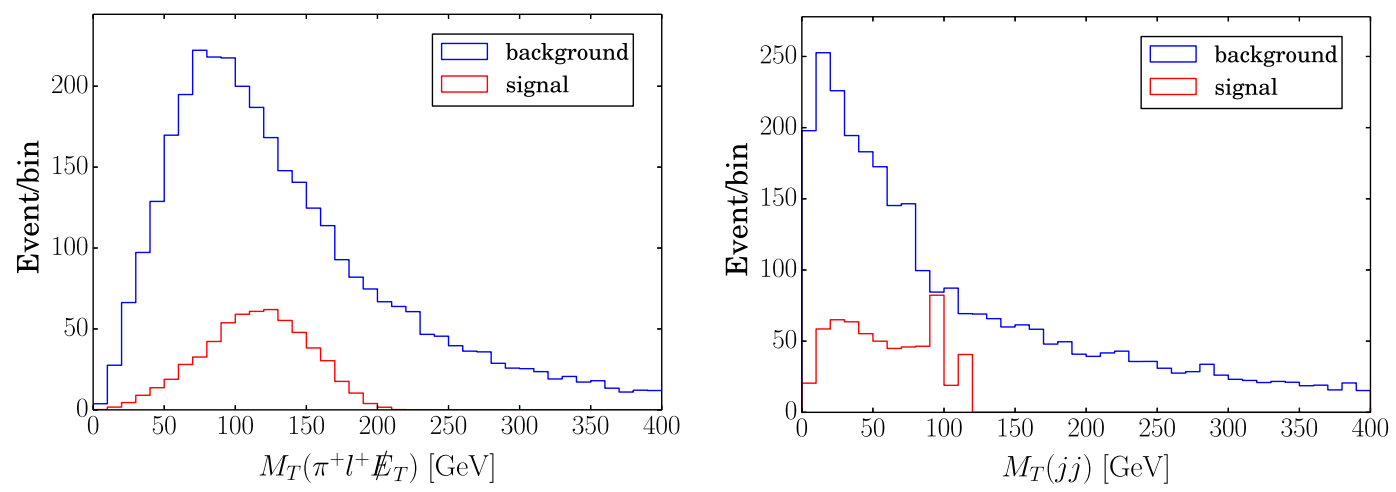

FIG. 6. The distribution of the signal and background events for $M_{T}\left(\pi^{+} \ell^{+} E_{T}\right)$ (the left figure) and $M_{T}(j j)$ (the right one) We use the basic cut in Eq. (29). The width of the bin in the figures is $10 \mathrm{GeV}$. We use the benchmark values in Eq. (32).

two jets, we can basically see twin Jacobian peaks at $m_{H_{1}}$ and $m_{H_{2}}$ [20]. Therefore, by using the distributions of $M_{T}\left(\tau^{+} \ell^{+} \mathscr{E}_{T}\right)$ and $M_{T}(j j)$, we can obtain the information on masses of all the charged scalars $H_{1}^{ \pm}, H_{2}^{ \pm}$, and $\Phi^{ \pm \pm}$. This is the characteristic feature of the process in this model. When we consider the decay of the tau lepton, the transverse mass of the decay products of the tau lepton and $\ell^{+} \nu \nu$ can be used instead of $M_{T}\left(\tau^{+} \ell^{+} \nu \nu\right)$.

In the following, we discuss the kinematics of the process at HL-LHC with the numerical evaluation. For input parameters, we take the following benchmark values as Scenario I;

$$
\begin{aligned}
m_{\Phi}=200 \mathrm{GeV}, & m_{H_{1}}=100 \mathrm{GeV}, \\
m_{H_{2}}=120 \mathrm{GeV}, & \tan \beta=10, \quad \chi=\frac{\pi}{4}, \\
m_{H}=130 \mathrm{GeV}, & m_{A}=140 \mathrm{GeV} .
\end{aligned}
$$

The doubly charged scalar $\Phi^{ \pm \pm}$is heavier than neutral scalars $H$ and $A$, and it can decay into final states including $H$ or $A$. However, the branching ratios of these decay channels are negligibly small because of small mass differences between $\Phi^{ \pm \pm}$and neutral scalars. Therefore, the discussions in Sec. III A are accurate in this scenario. In Appendix B 1, we show benchmark values of the scalar coupling constants to realize this mass spectrum, and we discuss the condition for the potential to be bounded from below.

In this scenario, we take large $\tan \beta(=10)$, so that they satisfy the constraints from flavor experiments $[42,43]$.
In addition, $H_{1,2}^{ \pm}$are heavier than the lower limit from the LEP $(80 \mathrm{GeV})$ [46]. At the LHC, $H_{1,2}^{ \pm}$in this scenario can be generated primarily via $t \bar{t}$ production. The cross section of this production process for $H_{1}^{ \pm}\left(H_{2}^{ \pm}\right)$is about 1.2 $\mathrm{pb}(0.73 \mathrm{pb})$ at $\sqrt{s}=13 \mathrm{TeV}$. The branching ratio of $H_{1}^{ \pm} \rightarrow \tau^{ \pm} \nu\left(H_{2}^{ \pm} \rightarrow \tau^{ \pm} \nu\right)$ is about 0.4. (See Sec. III A) Then, we obtain $\sigma_{H^{ \pm}} \times \operatorname{Br}\left(H_{1}^{ \pm} \rightarrow \tau \nu\right)=0.48 \mathrm{pb}$ and $\sigma_{H^{ \pm}} \times \operatorname{Br}\left(H_{2}^{ \pm} \rightarrow \tau \nu\right)=0.29 \mathrm{pb}$. Therefore, they satisfy the constraint from the LHC Run2 [47]. We also considered the constraints from the measurements of the oblique parameters [62] and the diphoton decay of the Higgs boson [63], and we found that this scenario satisfies both constraints. The details are shown in Appendix C and D.

The final state include the tau lepton, and we consider the case that the tau lepton decays into $\pi^{+} \bar{\nu}$. In this case, $\pi^{+}$ flies in the almost same direction of $\tau^{+}$in the center-ofmass (CM) frame because of the conservation of the angular momentum [53]. The branching ratio for $\tau^{+} \rightarrow$ $\pi^{+} \bar{\nu}$ is about $11 \%$ [62], and we assume that the efficiency of tagging the hadronic decay of tau lepton is $60 \%$ [64]. Under the above setup, we carry out the numerical evaluation of the signal events by using MadGraph5_aMC@NLO [60], FEYNRULES [61], and TauDecay [65]. As a result, about 600 signal events are expected to be produced at HL-LHC. The distributions of the signal events for $M_{T}\left(\pi^{+} \ell^{+} \mathscr{E}_{T}\right)$ and $M_{T}(j j)$ are shown in red line in the left figure of Fig. 6 and in the right one, respectively.

Next, we discuss the background events and their reduction. The main background process is $p p \rightarrow W^{+} W^{+} j j \rightarrow$ $\tau^{+} \ell^{+} \nu \bar{\nu} j j$. The leading order of this background process is

TABLE II. Numbers of signal event and background events at HL-LHC in Scenario I. In the first column, the number of events under only the basic cuts are shown. The number of events under the all cuts are shown in the second column. We use the benchmark values in Eq. (32).

\begin{tabular}{lccc}
\hline \hline & Signal $S$ & Background $B$ & $S / \sqrt{S+B}$ \\
\hline Basic cuts Eq. (29) & 592 & 3488 & 9.3 \\
Basic cuts [Eq. (29)] and $\Delta R_{j j}<2,\left|\Delta \eta_{j j}\right|<2.5$ & 487 & 412 & 16 \\
All cuts Eq. (29) and Eq. (33) & 487 & 75 & 20 \\
\hline \hline
\end{tabular}



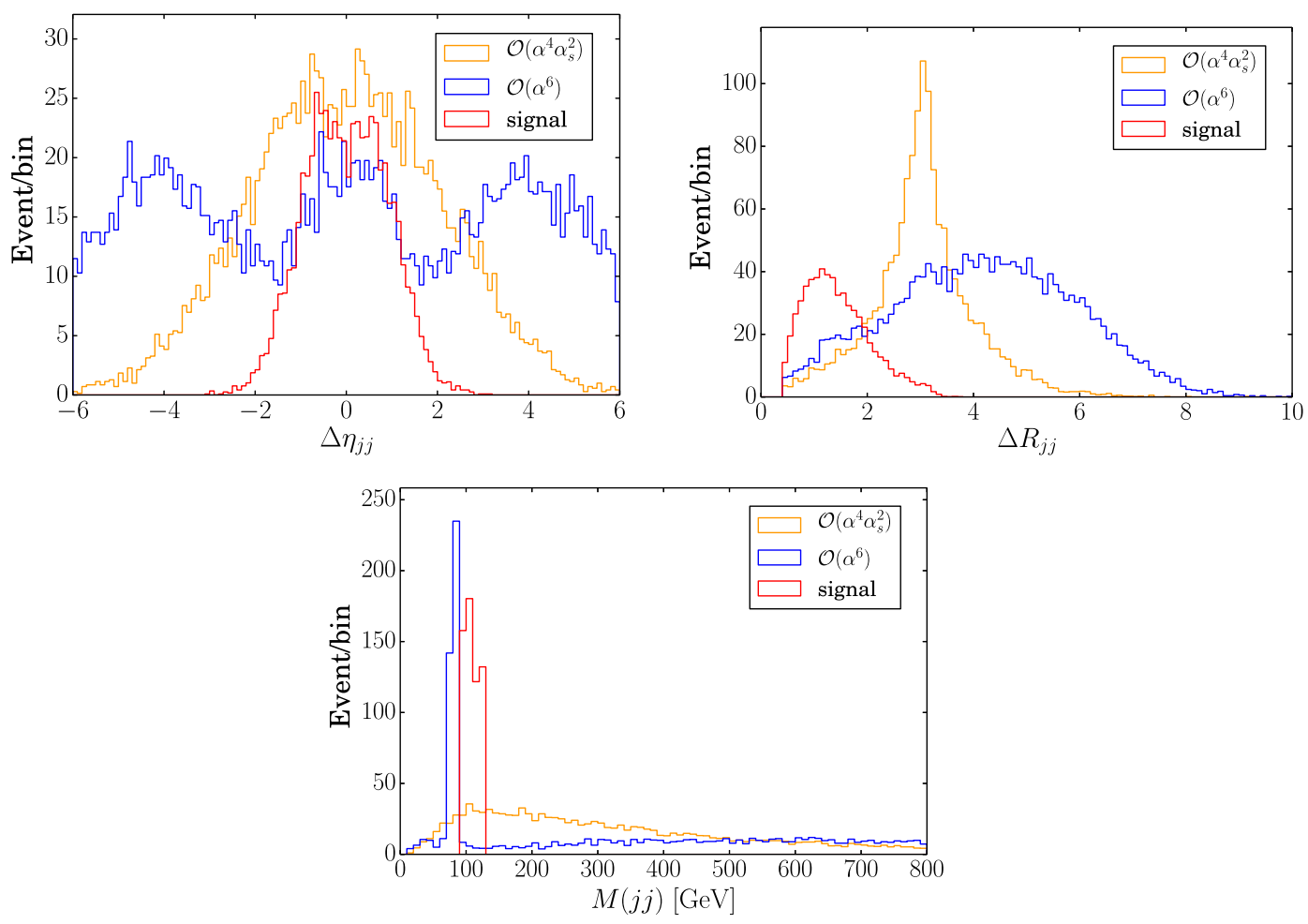

FIG. 7. The distributions of signal and background events for $\Delta \eta_{j j}$ (the upper left figure), $\Delta R_{j j}$ (the upper right one), and $M_{j j}$ (the bottom one). The red lines are those for the signal events. The blue (yellow) lines are those for the background events of $\mathcal{O}\left(\alpha^{6}\right)$ $\left(\mathcal{O}\left(\alpha^{4} \alpha_{s}^{2}\right)\right)$. In the figures for $\Delta \eta_{j j}$ and $\Delta R_{j j}$, we take the width of bins as 0.1 . In the figure for $M_{j j}$, the width of bins is $10 \mathrm{GeV}$. We use the benchmark values in Eq. (32).

$\mathcal{O}\left(\alpha^{6}\right)$ and $\mathcal{O}\left(\alpha^{4} \alpha_{s}^{2}\right)$. For $\mathcal{O}\left(\alpha^{6}\right)$, the vector boson fusion (VBF) and tri-boson production $p p \rightarrow W^{+} W^{+} W^{-} \rightarrow$ $W^{+} W^{+} j j$ are important. On the other hand, for $\mathcal{O}\left(\alpha^{4} \alpha_{s}^{2}\right)$, the main process is t-channel gluon mediated $p p \rightarrow$ $q^{*} q^{\prime *} \rightarrow W^{+} W^{+} j j$, where $q$ and $q^{\prime}$ are quarks in internal lines. The number of the total background events under the basic cuts in Eq. (29) is shown in Table II. Transverse mass distributions of background events for $M_{T}\left(\pi^{+} \ell^{+} E_{T}\right)$ and $M_{T}(j j)$ are shown in the blue line in the left figure of Fig. 6 and in the right one, respectively. The number of the background events is larger than that of the signal. Clearly, background reduction has to be performed by additional kinematical cuts. We give some comments about other background processes in the end of this subsection.

First, we impose the pseudorapidity cut for a pair of two jets $\left(\Delta \eta_{j j}\right)$. The $\Delta \eta_{j j}$ distributions of the signal and background processes are shown in the upper left figure in Fig. 7. For the signal events, the distribution has a maximal value at $\Delta \eta_{j j}=0$ as they are generated via the decay of $H_{1}^{-}$ or $H_{2}^{-}$. On the other hand, for the VBF background, two jets fly in the almost opposite directions, and each jet flies almost along the beam axis. Large $\left|\Delta \eta_{j j}\right|$ is then expected to appear [66], so that we can use $\left|\Delta \eta_{j j}\right|<2.5$ to reduce the VBF background. We note that this kinematical cut is not so effective to reduce other $\mathcal{O}\left(\alpha^{6}\right)$ and $\mathcal{O}\left(\alpha^{4} \alpha_{s}^{2}\right)$ processes because in these background, the distribution are maximal at $\Delta \eta_{j j}=0$.

Second, we impose the angular distance cut for a pair of two jets $\left(\Delta R_{j j}\right)$. The $\Delta R_{j j}$ distributions of the signal and background processes are shown in the upper right figure in Fig. 7. For the signal events, the distribution has a maximal value at $\Delta R_{j j} \simeq 1.0$. On the other hand, for the $\mathcal{O}\left(\alpha^{4} \alpha_{s}^{2}\right)$ background events, $\Delta R_{j j}$ has a peak at $\Delta R_{j j} \sim \pi$. In addition, in the $\mathcal{O}\left(\alpha^{6}\right)$ ones, $\Delta R_{j j}$ has large values between 3 and 6. Therefore, for $\Delta R_{j j}<2$, the background events are largely reduced while the almost all signal events remains.

Third, we impose invariant mass cut for a pair of two jets $\left(M_{j j}\right)$. The $M_{j j}$ distributions of the signal and background processes are shown in the bottom figure in Fig. 7. For the signal events, as they are generated via the decay of the singly charged scalars, the distribution has twin peaks at the masses of $H_{1}^{ \pm}$and $H_{2}^{ \pm}(100 \mathrm{GeV}$ and $120 \mathrm{GeV})$. On the other hand, for the background events, the jets are generated via on-shell $W$ or t-channel diagrams. Then, the distribution of the background has a peak at the $W$ boson mass $(\sim 80 \mathrm{GeV})$. Thus, the kinematical cut $90 \mathrm{GeV}<$ $M_{j j}<180 \mathrm{GeV}$ is so effective to reduce the background events. We note that this reduction can only be possible when we already know some information on the masses of the singly charged scalars. 

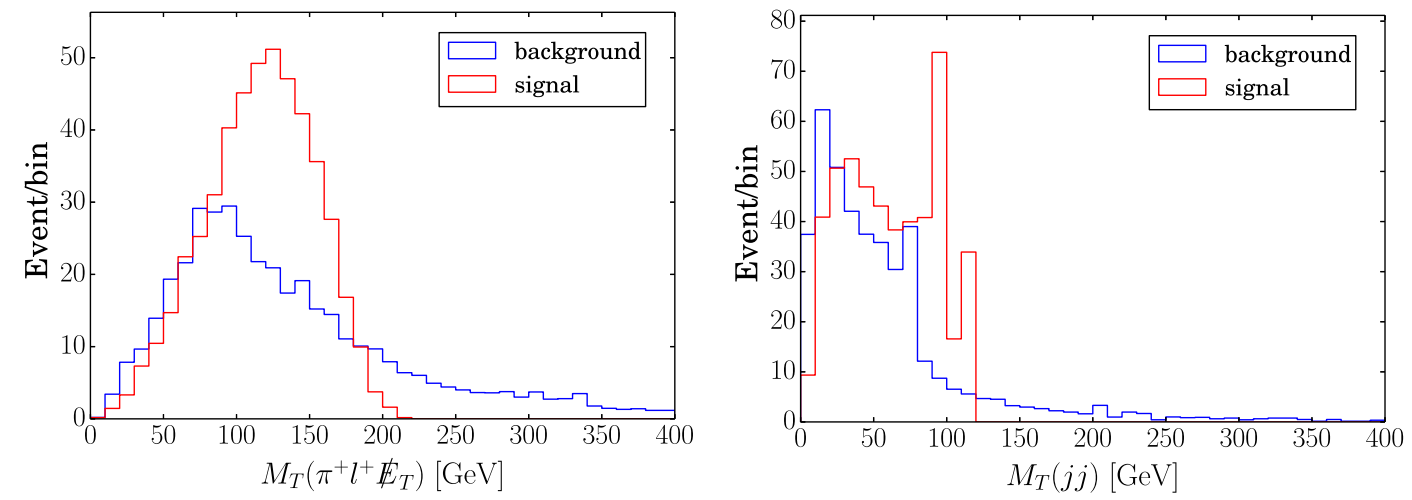

FIG. 8. The distribution of the signal and background events for $M_{T}\left(\pi^{+} \ell^{+} \boldsymbol{E}_{T}\right)$ (the left figure) and $M_{T}(j j)$ (the right one) We use the basic cuts in Eq. (29), $\left|\Delta \eta_{j j}\right|<2.5$, and $\Delta R_{j j}<2$. The width of bins in the figures is $10 \mathrm{GeV}$. We use the benchmark values in Eq. (32).

We summarize three kinematical cuts for the background reduction.

(i) $\left|\Delta \eta_{j j}\right|<2.5$,

(ii) $\Delta R_{j j}<2$,

(iii) $90 \mathrm{GeV}<M_{j j}<180 \mathrm{GeV}$,

Let us discuss how the backgrounds can be reduced by using the first two kinematical cuts (i) and (ii), in addition to the basic cuts given in Eq. (29). This corresponds to the case that we do not use the information on the masses of the singly charged scalars. The results are shown in the third column of Table II. In this case, about $88 \%$ of the background events are reduced, while about $82 \%$ of the signal events remain. We obtain the significance as $S / \sqrt{S+B}=16$. The distributions for $M_{T}\left(\pi^{+} \ell^{+} E_{T}\right)$ and $M_{T}(j j)$ are shown in Fig. 8. In the left figure of Fig. 8, we can see the Jacobian peak of $M_{T}\left(\pi^{+} \ell^{+} \mathscr{E}_{T}\right)$. Consequently, the signal process can be detected at HL-LHC in Scenario I of Eq. (32). However, the endpoint of the signal is unclear due to the background events, so that it would be difficult to precisely decide the mass of $\Phi^{++}$. On the other hand, we can see the twin Jacobian peaks of $M_{T}(j j)$ in the right figure of Fig. 8. Therefore, we can also obtain information on masses of both the singly charged scalars. In this way, all the charged scalar states $\Phi^{ \pm \pm}, H_{1}^{ \pm}$, and $H_{2}^{ \pm}$can be detected and their masses may be obtained to some extent.

Furthermore, if we impose all the kinematical cuts (i), (ii), and (iii) with the basic cuts, the backgrounds can be further reduced. The results are shown in the fourth column of Table II. The number of signal events are same with that in the previous case. On the other hand, the background reduction is improved, and 98\% of the background events are reduced. The significance is also improved as $S / \sqrt{S+B}=20$. Distributions for $M_{T}\left(\pi^{+} \ell^{+} \mathscr{E}_{T}\right)$ and $M_{T}(j j)$ are shown in Fig 9. In the left figure of Fig 9, we can see that there are only few background events around the end point of Jacobian peak $M_{T}\left(\pi^{+} \ell^{+} \mathscr{E}_{T}\right)$. Thus, it would be expected we obtain the more clear information on $m_{\Phi}$ than that from the case where only (i) and (ii) are imposed as additional kinematical cuts. We can also clearly see the twin Jacobian peaks in the right figure of Fig. 9, and a large improvement can be achieved for the determination of the masses of both the singly charged scalar states.
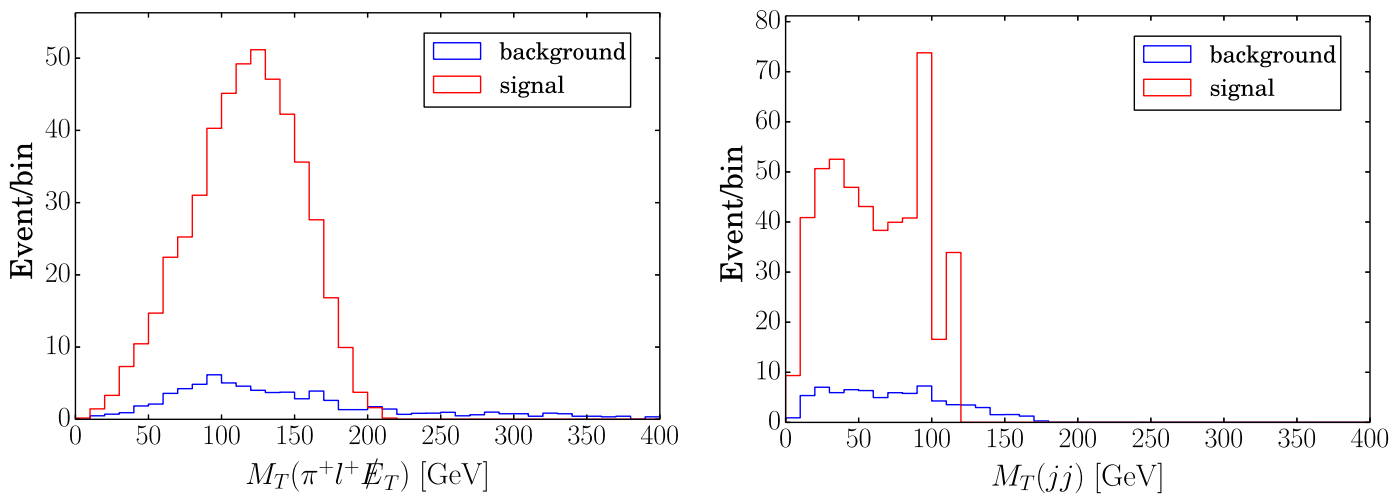

FIG. 9. The distribution of the signal and background events for $M_{T}\left(\pi^{+} \ell^{+} \boldsymbol{E}_{T}\right)$ (the left figure) and $M_{T}(j j)$ (the right figure) We use the basic cut in Eq. (29) and all the kinematical cuts in Eq. (33). The width of the bin in the figures is $10 \mathrm{GeV}$. 
Now, we give a comment about the detector resolution. In the process, the transverse momenta of jets $\left(p_{T}^{j}\right)$ are mainly distributed between 0 and $200 \mathrm{GeV}$, and the typical value of them is about $100 \mathrm{GeV}$. According to Ref. [67], at the current ATLAS detector, the energy resolution for $p_{T}^{j} \simeq 100 \mathrm{GeV}$ is about $10 \%$. In Figs. 6-9, we take the width of bins as $10 \mathrm{GeV}$. Therefore, it would be possible that the twin Jacobian peaks in the distribution for $M_{T}(j j)$ overlap each other and they looks like one Jacobian peak with the unclear endpoint at the ATLAS detector if the mass differences is not large enough. Then, it would be difficult to obtain the information on both $m_{H_{1}}$ and $m_{H_{2}}$ from the transverse momentum distribution. Even in this case, it would be able to obtain the hint for the masses by investigating the process.

Before closing Sec. IVA, we briefly discuss other SM background processes. In our analysis, we did not consider the background where the $Z$ boson decays into dijet such as $q q \rightarrow Z^{*} \rightarrow Z h \rightarrow j j \tau \bar{\tau} \rightarrow j j \pi^{+} \bar{\nu}_{\tau} \ell^{-} \nu_{\tau} \bar{\nu}_{\ell}$, which can be expected to be reduced by veto the events of $M_{j j}$ at the $Z$ boson mass and the cut of the transverse mass $M_{T}\left(\pi^{+} \ell^{+} \mathscr{E}_{T}\right)$ below $125 \mathrm{GeV}$. It does not affect the Jacobian peak and the endpoint at the mass of doubly charged scalar boson $\Phi^{ \pm \pm}$. In addition, in the above analyses, we did not consider the background from other vector boson fusion processes $Z W j j$ and $Z Z j j$. These processes generate final states similar to that of the signal;

$$
\begin{gathered}
p p \rightarrow W^{+} Z j j \rightarrow \tau^{+} \tau^{-} \ell^{+} \nu_{\ell} j j, \\
p p \rightarrow W^{+} Z j j \rightarrow \tau^{+} \tau^{-} \ell^{+} \ell^{-} \nu_{\tau} j j, \\
p p \rightarrow Z Z j j \rightarrow \ell^{+} \ell^{-} \nu \bar{\nu} j j, \\
p p \rightarrow Z Z j j \rightarrow \tau^{+} \tau^{-} \nu \bar{\nu} j j \rightarrow \tau^{+} \ell^{-} \nu_{\tau} \bar{\nu}_{\ell} \nu \bar{\nu} j j .
\end{gathered}
$$

These processes generate the same final state with that of the signal $\left(\tau^{+} \ell^{+} j j E\right)$ with misidentifications at detectors, for example the charge misidentification of leptons [68], misidentifying isolated leptons as candidates of hadronic decays of $\tau$ [64], and so on. Considering rates of these misidentifications, the expected numbers of these processes at the $\operatorname{HL}-\mathrm{LHC}\left(\sqrt{s}=14 \mathrm{TeV}, \mathcal{L}=3000 \mathrm{fb}^{-1}\right)$ are $\mathcal{O}(1)$ under the kinematical cuts in Eqs. (29) and (33). Therefore, it is concluded that these background processes do not affect the Jacobian peaks in the signal events.

\section{B. Scenario II}

In this scenario, the singly charged scalars predominantly decay into $t b$ with the branching ratio almost $100 \%$. We investigate the signal $p p \rightarrow W^{+*} \rightarrow \Phi^{++} H_{1,2}^{-} \rightarrow$ $t \bar{t} b \bar{b} \ell^{+} \nu \rightarrow b b \bar{b} \bar{b} \ell^{+} \ell^{\prime+} \nu \nu j j\left(\ell, \ell^{\prime}=e, \mu\right)$. The Feynman diagram for the process is shown in Fig. 10. The decay products of $\Phi^{++}$and $H_{1,2}^{ \pm}$are $b \bar{b} \ell^{+} \ell^{\prime+} \nu \nu$ and $b \bar{b} j j$,

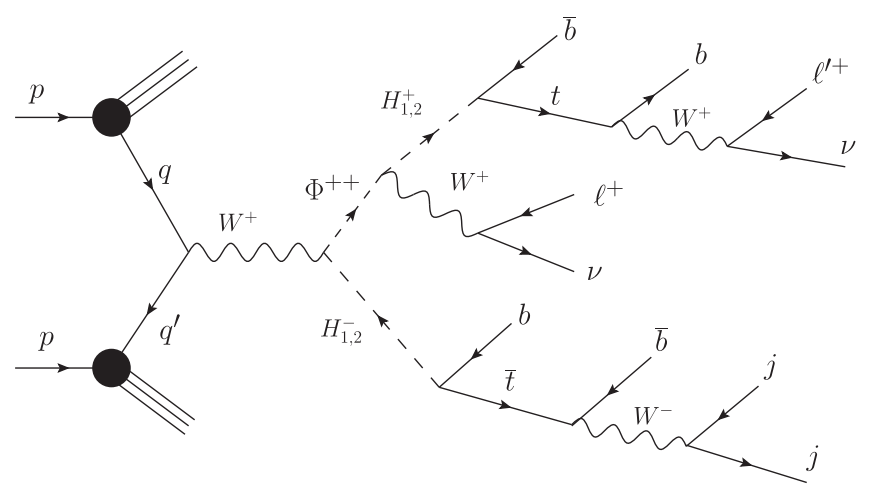

FIG. 10. The Feynman diagram for the signal process in Scenario II, where $q$ and $q^{\prime}$ are partons.

respectively. Therefore, in the same way as Scenario I, we can obtain information on masses of all the charged scalars by investigating the transverse distributions of signal and background events for $M_{T}\left(b \bar{b} \ell^{+} \ell^{\prime+} \nu \nu\right)$ and $M_{T}(b \bar{b} j j)$. However, in the Scenario II, decay products of both $\Phi^{++}$ and $H_{1,2}^{-}$include a $b \bar{b}$ pair, and it is necessary to distinguish the origin of the two $b \bar{b}$ pairs. We suggest the following two methods of the distinction.

In the first method, we use the directions of $b$ and $\bar{b}$. In the process, $\Phi^{++}$and $H_{1,2}^{-}$are generated with momenta in the opposite directions, and decay products fly along the directions of each source particle. The both of two $W$ bosons generated via the decay of $\Phi^{++}$decay into charged leptons and neutrinos, while the $W$ boson via the decay of $H_{1,2}$ decays into a pair of jets. By using this topology of the process, we can distinguish the origin of two $b \bar{b}$ pairs. The $b \bar{b}$ pair which flies along the charged leptons $\ell^{+}$and $\ell^{\prime+}$ (and flies along the almost opposite direction of a pair of jets) comes from the decay of $\Phi^{++}$. The other $b \bar{b}$ pair is the decay product of $H_{1,2}^{-}$.

In the second method, we use the transverse momenta of $b$ and $\bar{b}$. As shown in the Feynman diagram in Fig. 10, in the decay chain of $\Phi^{++}, b$ is generated via the decay of the top quark while $\bar{b}$ is generated via the decay of the singly charged scalars from the decay of $\Phi^{++}$. On the other hand, in the decay chain of $H_{1,2}^{-}, b$ is generated via the decay of the singly charged scalars while $\bar{b}$ is generated via the decay of the antitop quark. Therefore, when the singly charged scalars are heavy enough to satisfy the inequality,

$$
m_{H_{1,2}}-m_{t}-m_{b}>m_{t}-m_{W}-m_{b},
$$

the typical value of the transverse momentum of $b$ from $H_{1,2}^{-}$is larger than that of $b$ from the top quark. In the same way, the typical value of transverse momentum of $\bar{b}$ from $H_{1,2}^{+}$is larger than that of $\bar{b}$ from the antitop quark. Therefore, in this case, we can construct the $b \bar{b}$ pair which mainly comes from the decay of $\Phi^{++}$by selecting $b$ with the smaller transverse momentum and $\bar{b}$ with the larger 
transverse momentum. The other $b \bar{b}$ pair comes from the decay of $H_{1,2}^{-}$. On the contrary, when the singly charged scalars are light enough to satisfy the inequality,

$$
m_{H_{1,2}}-m_{t}-m_{b}<m_{t}-m_{W}-m_{b},
$$

the typical value of the transverse momentum of $b(\bar{b})$ from $H_{1,2}^{-}\left(H_{1,2}^{+}\right)$is smaller than that of $b(\bar{b})$ from the top quark (the antitop quark). Therefore, in the case where the singly charged scalar is so light that they satisfy the inequality in Eq. (41), we can construct the $b \bar{b}$ pair which mainly comes from the decay of $\Phi^{++}$by selecting $b$ with the larger transverse momentum and $\bar{b}$ with the smaller transverse momentum. The other $b \bar{b}$ pair comes from the decay of $H_{1,2}^{-}$. Finally, when the masses of singly charged scalars are around $250 \mathrm{GeV}$, they satisfy the equation,

$$
m_{H_{1,2}}-m_{t}-m_{b} \simeq m_{t}-m_{W}-m_{b}
$$

Then, the typical values of the transverse momenta of two $b$ are similar, and those of two $\bar{b}$ are also similar. Therefore, we can construct the correct $b \bar{b}$ pair only partly by using the above method, and it is not so effective. In this case, the first method explained in the previous paragraph is needed.

In the following, we discuss the signal and the background events at HL-LHC with the numerical calculation. In the numerical evaluation, we take the following benchmark values as Scenario II;

$$
\begin{aligned}
m_{\Phi}=300 \mathrm{GeV}, & m_{H_{1}}=200 \mathrm{GeV}, \\
m_{H_{2}}=250 \mathrm{GeV}, & \tan \beta=3, \quad \chi=\frac{\pi}{4}, \\
m_{H}=210 \mathrm{GeV}, & m_{A}=220 \mathrm{GeV} .
\end{aligned}
$$

In this scenario, $\Phi^{ \pm \pm}$and $H_{2}^{ \pm}$are heavier than neutral scalars $H$ and $A$, and they can decay into final states including $H$ or $A$. However, the branching ratios of these channels are negligibly small because of the small mass differences between charged scalars and neutral scalars. Therefore, the discussions in Sec. III A are accurate in this scenario. In Appendix B 2, we show benchmark values of the scalar coupling constants to realize this mass spectrum, and we discuss the condition for the potential to be bounded from below.

In this scenario, we assume that $\tan \beta=3$ so that they satisfy the constraints from flavor experiments $[42,43]$. In addition, $H_{1,2}^{ \pm}$are heavier than the lower limit from the LEP $(80 \mathrm{GeV})$ [46]. At the LHC, $H_{1,2}^{ \pm}$in this scenario can be generated primarily by associate production with $t b\left(g g \rightarrow H_{1,2}^{ \pm} t b\right)$. The cross section of this production process for $H_{1}^{ \pm}\left(H_{2}^{ \pm}\right)$is about $0.12 \mathrm{pb}(0.073 \mathrm{pb})$ at $\sqrt{s}=13 \mathrm{TeV}$. The singly charged scalars $H_{1,2}^{ \pm}$decay into $t b$ at almost $100 \%$. (See Sec. III A) Then, we obtain $\sigma_{H^{ \pm}} \times$ $\operatorname{Br}\left(H_{1}^{ \pm} \rightarrow t b\right) \simeq 0.12 \mathrm{pb}$ and $\sigma_{H^{ \pm}} \times \operatorname{Br}\left(H_{2}^{ \pm} \rightarrow t b\right) \simeq 0.073 \mathrm{pb}$.
TABLE III. Numbers of signal event and background events under the basic cuts in Eq. (29) in Scenario II. We assume that the efficiency of $b$-tagging is $70 \%$. We use the benchmark values in Eq. (43).

\begin{tabular}{lccc}
\hline \hline & Signal $S$ & Background $B$ & $S / \sqrt{S+B}$ \\
\hline Basic cuts (Eq. (29)) & 145 & 40 & 11 \\
\hline \hline
\end{tabular}

Therefore, they satisfy the constraint from the LHC Run2 [48]. We also have considered the constraints from the measurements of the oblique parameters [62] and the measurement of the diphoton decay of the Higgs boson at the LHC [63], and we have found that this scenario satisfies both of them. The details are shown in Appendix C and D.

In addition, we adopt the assumption about the collider performance at HL-LHC in Eq. (28), and we use the basic kinematical cuts in Eq. (29). The final state of the signal includes two bottom quarks and two antibottom quarks, and we assume that the efficiency of the $b$-tagging is $70 \%$ per one bottom or antibottom quark [69]. Thus, the total efficiency of the $b$-tagging in the signal event is about $24 \%$. In the numerical calculation, we use MadGraph5_aMC@NLO [60], FEYNRULES [61].

As a result, 145 events are expected to appear at HL-LHC as shown in Table III. In this benchmark scenario of Eq. (43), $H_{1}^{ \pm}$is so light that we can use the distinction of the $b \bar{b}$ pair in the case where $m_{H_{1}}-m_{t}-m_{b}<m_{t}-$ $m_{b}-m_{W}$. Therefore, we can construct the $b \bar{b}$ pair which mainly comes from the decay of $H_{1}^{-}$by selecting $b$ with the smaller transverse momentum and $\bar{b}$ with the larger transverse momentum. On the other hand, the mass of $H_{2}^{ \pm}$is $250 \mathrm{GeV}$, and it satisfies the equation $m_{H_{2}}-m_{t}-m_{b} \simeq m_{t}-m_{b}-m_{W}$. Therefore, the selection of $b$ and $\bar{b}$ by their transverse momenta is partly effective in the signal where $H_{2}^{-}$is produced with $\Phi^{++}$via $W^{+*}$. ${ }^{9}$

In Figs. 11, we show the distributions of $M_{T}\left(b_{1} \bar{b}_{2} \ell^{+} \ell^{\prime+} \boldsymbol{E}_{T}\right)$ and $M_{T}\left(b_{2} \bar{b}_{1} j j\right)$, where $b_{1}\left(\bar{b}_{1}\right)$ is the bottom quark (anti-bottom quark) with the larger transverse momentum and $b_{2}\left(\bar{b}_{2}\right)$ is the other. In the left figure of Fig. 11, the endpoint of the Jacobian peak is not so sharp because the selection of the $b \bar{b}$ pairs do not work well in the associated production of $\Phi^{++}$and $H_{2}^{-}$. In the right figure of Fig. 11, we can see the twin Jacobian peaks at the masses of the singly charged scalars. However, the number of events around the Jacobian peaks, especially the one due to $H_{2}^{ \pm}$, are small, and it would be difficult to obtain information on masses form the distribution for $M_{T}\left(b_{2} \bar{b}_{1} j j\right)$. In order to obtain the clearer information on $m_{H_{1,2}}$, we can use the invariant mass of $b_{2} \bar{b}_{1} j j$ instead of $M_{T}\left(b_{2} \bar{b}_{1} j j\right)$.

\footnotetext{
${ }^{9}$ We note that we assume some information on the mass of singly charged scalars to select the kinematical cuts.
} 

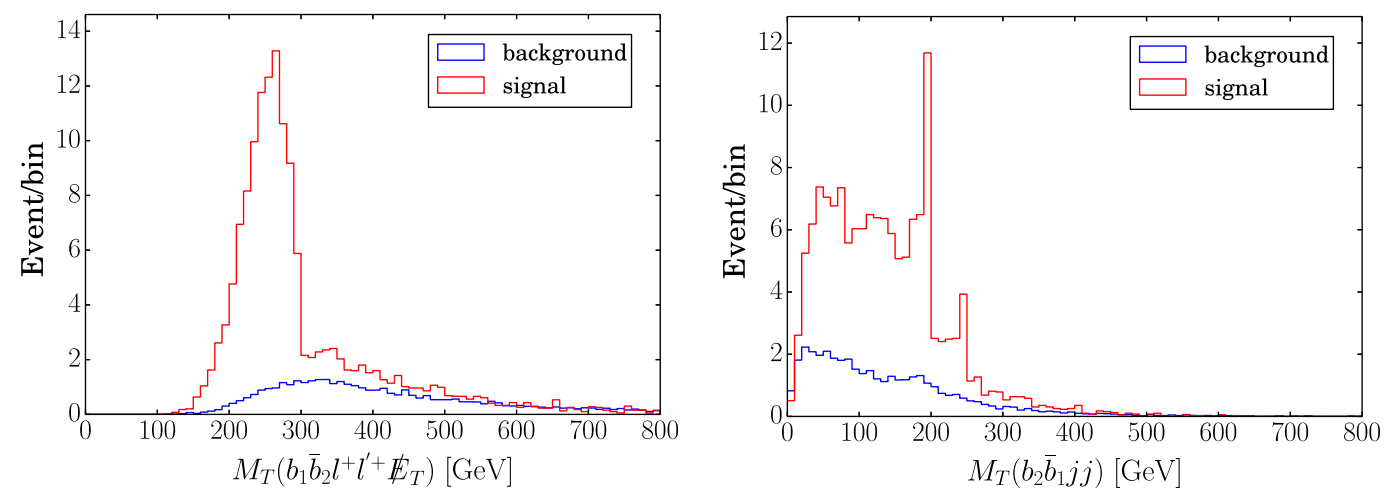

FIG. 11. The distribution of $M_{T}\left(b_{1} \bar{b}_{2} \ell^{+} \ell^{\prime+} E_{T}\right)$ (the left one) and $M_{T}\left(b_{2} \bar{b}_{1} j j\right)$ (the right one) in the signal and background events under the kinematical cuts in Eq. (29). In the figures, the width of bins is $10 \mathrm{GeV}$. We use the benchmark values in Eq. (43).

In Fig. 12, we show the distributions of signal and backgrounds for the invariant mass of $b_{2} \bar{b}_{1} j j$. The numbers of events at the twin peaks are $\mathcal{O}(30)$ and $\mathcal{O}(10)$, which are larger than thaose at the twin Jacobian peaks in the figure for $M_{T}\left(b_{2} \bar{b}_{1} j j\right)$ (the right figure of Fig 11).

Next, we discuss the background events at HL-LHC. We consider the process $p p \rightarrow t \bar{t} b \bar{b} W^{+} \rightarrow b b \bar{b} \bar{b} W^{+} W^{+} W^{-} \rightarrow$ $b b \bar{b} \bar{b} \ell^{+} \ell^{\prime+} \nu \nu j j$ as the background. As a result of the numerical calculation, 40 events are expected to appear at HL-LHC as shown in Table III. This is the same order with the signal events. In Fig. 11, the distributions of $M_{T}\left(b_{1} \bar{b}_{2} \ell^{+} \ell^{\prime+} E_{T}\right)$ and $M_{T}\left(b_{2} \bar{b}_{1} j j\right)$ in the background events are shown. We use only the basic cuts in Eq. (29) in the numerical calculation. Nevertheless, in the both figures of Fig. 11, the number of signal events around the Jacobian peaks are much larger than those of the background events.

In Fig. 12, the distribution of the background events for the invariant mass $M\left(b_{2} \bar{b}_{1} j j\right)$ in the background events are shown. The numbers of signal events around the two peaks are much larger than those of the background events.

In summary, it would be possible that we obtain information on masses of all the charged scalars $H_{1}^{ \pm}$, $H_{2}^{ \pm}$, and $\Phi^{ \pm \pm}$by investigating the transverse mass

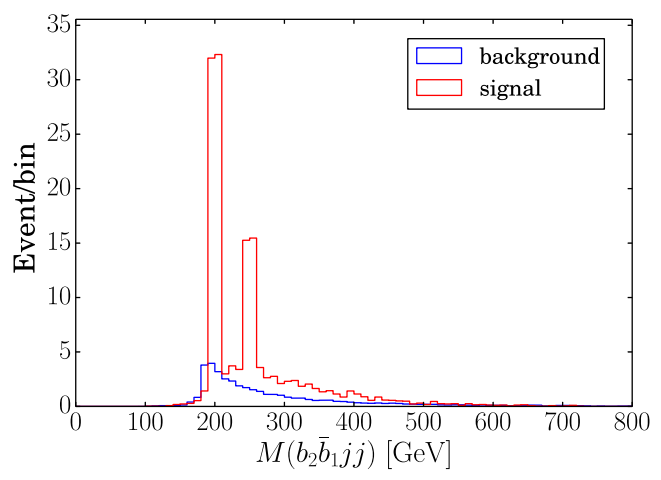

FIG. 12. The distribution of the invariant mass of $b_{2} \bar{b}_{1} j j$ in the signal and background events under the kinematical cuts in Eq. (29). In the figure, the width of bins is $10 \mathrm{GeV}$. We use the benchmark values in Eq. (43). distribution for $M_{T}\left(b_{2} \bar{b}_{1} \ell^{+} \ell^{\prime+} \mathcal{E}_{T}\right)$ and $M_{T}\left(b_{1} \bar{b}_{2} j j\right)$ and the invariant mass distribution for $M\left(b_{1} \bar{b}_{2} j j\right)$ at HL-LHC.

Before closing subsection $\mathrm{B}$, we give a comment about the detector resolution. In the process of Scenario II, the typical value of the transverse momenta of jets and bottom quarks is about $100 \mathrm{GeV}$. As mentioned in the end of the section for Scenario I, at the ATLAS detector, the energy resolution for $p_{T}^{j} \simeq 100 \mathrm{GeV}$ is about $10 \%$ [67]. In Figs. 11 and 12 , we take the width of bins as $10 \mathrm{GeV}$. Therefore, it would be possible that the twin Jacobian peaks in the distribution for $M_{T}(j j)$ or $M(j j)$ overlap each other and they looks like one Jacobian peak with the unclear endpoint at the ATLAS detector if the mass differences is not large enough. Then, it would be difficult to obtain the information on both $m_{H_{1}}$ and $m_{H_{2}}$ from the transverse momentum distribution. Even in this case, it would be able to obtain the hint for masses by investigating the process.

\section{SUMMARY AND CONCLUSION}

We have investigated collider signatures of the doubly and singly charged scalar bosons at the HL-LHC by looking at the transverse mass distribution as well as the invariant mass distribution in the minimal model with the isospin doublet with the hypercharge $Y=3 / 2$. We have discussed the background reduction for the signal process $p p \rightarrow W^{+*} \rightarrow$ $\Phi^{++} H_{1,2}^{-}$in the following two cases depending on the mass of the scalar bosons with the appropriate kinematical cuts. (1) The main decay mode of the singly charged scalar bosons is the tau lepton and missing (as well as charm and strange quarks). (2) That is into a top bottom pair. In the both cases, we have assumed that the doubly charged scalar boson is heavier than the singly charged ones. It has been concluded that the scalar doublet field with $Y=3 / 2$ is expected to be detectable for these cases at the HL-LHC unless the masses of $\Phi^{ \pm \pm}$and $H_{1,2}^{ \pm}$are too large.

\section{ACKNOWLEDGMENTS}

We would like to thank Arindam Das and Kei Yagyu for useful discussions. This work is supported by Japan 
Society for the Promotion of Science, Grant-in-Aid for Scientific Research, No. 16H06492, No. 18F18022, No. 18F18321, and No. 20H00160.

\section{APPENDIX A: SOME FORMULAS FOR THE DECAYS OF CHARGED SCALARS}

In this section, we show some analytic formulae for decay rates of the charged scalars $H_{1,2}^{ \pm}$and $\Phi^{ \pm \pm}$.

\section{Formulas for decays of the singly charged scalars $\boldsymbol{H}_{1,2}^{ \pm}$}

\section{a. 2-body decays}

The decay rate for the decay of $H_{i}^{ \pm}(i=1,2)$ into a pair of quarks $q q^{\prime}$ is given by

$$
\begin{aligned}
\Gamma\left(H_{i}^{ \pm} \rightarrow q q^{\prime}\right)= & \frac{3 m_{H_{i}}}{8 \pi}\left(\frac{m_{H_{i}}^{2}}{v^{2}}\right) \chi_{i}^{\prime 2} \cot ^{2} \beta\left|V_{q q^{\prime}}\right|^{2}\left(\left(r_{q}+r_{q^{\prime}}\right)\right. \\
& \left.-\left(r_{q}+r_{q^{\prime}}\right)^{2}-4 r_{q} r_{q^{\prime}}\right) F\left(r_{q}, r_{q^{\prime}}\right),
\end{aligned}
$$

where $r_{q}\left(r_{q^{\prime}}\right)$ is the ratio of the squared mass of quark $q$ $\left(q^{\prime}\right)$ to the squared mass of $H_{i}^{ \pm}$:

$$
r_{q}=\frac{m_{q}^{2}}{m_{H_{i}}^{2}}, \quad r_{q^{\prime}}=\frac{m_{q^{\prime}}^{2}}{m_{H_{i}}^{2}},
$$

and $\chi_{i}^{\prime}$ is defined as follows;

$$
\chi_{1}^{\prime}=\cos \chi, \quad \chi_{2}^{\prime}=\sin \chi .
$$

The function $F(x, y)$ in Eq. (A1) is defined as

$$
F(x, y)=\sqrt{1+(x-y)^{2}-2(x+y)} .
$$

The decay rate for the decay of $H_{i}^{ \pm}$into a charged lepton $\ell$ and a neutrino $\nu_{\ell}$ is given by

$\Gamma\left(H_{i}^{ \pm} \rightarrow \ell \nu_{\ell}\right)=\frac{m_{H_{i}}}{8 \pi}\left(\frac{m_{\ell}}{v}\right)^{2} \chi_{i}^{\prime 2} \cot ^{2} \beta\left(1-\frac{m_{\ell}^{2}}{m_{H_{i}}^{2}}\right)$,

where $m_{\ell}$ is mass of $\ell$.

In the case that $m_{H_{i}}>m_{H_{j}}+m_{Z}(i, j=1,2, i \neq j)$, the decay $H_{i}^{ \pm} \rightarrow H_{j}^{ \pm} Z$ is allowed, and its decay rate is given by

$$
\Gamma\left(H_{i} \pm \rightarrow H_{j}^{ \pm} Z\right)=\frac{m_{H_{i}}}{16 \pi}\left(\frac{m_{H_{i}}}{v}\right)^{2} \sin ^{2} 2 \chi F\left(r_{Z}, r_{j}\right)^{3} \quad(i \neq j),
$$

where

$$
r_{Z}=\frac{m_{W}^{2}}{m_{H_{i}}^{2}}, \quad r_{j}=\frac{m_{H_{j}}^{2}}{m_{H_{i}}^{2}} .
$$

\section{b. 3-body decays}

The decay rate for $H_{i}^{ \pm} \rightarrow t^{*} b \rightarrow W^{ \pm} b \bar{b}$ is given by

$$
\Gamma\left(H_{i}^{ \pm} \rightarrow t^{*} b \rightarrow W^{ \pm} b \bar{b}\right)=\frac{3 m_{H_{i}}}{128 \pi^{3}}\left(\frac{m_{t}}{v}\right)^{4} \chi_{i}^{\prime 2} \cot ^{2} \beta\left|V_{t b}\right|^{2} \int_{r_{W}}^{1} \frac{\mathrm{d} x}{x} \frac{(1-x)^{2}\left(x-r_{W}\right)^{2}\left(x+2 r_{W}\right)}{\left(x-r_{t}\right)^{2}+r_{t} r_{\Gamma_{t}}},
$$

where mass of the bottom quark is neglected, and $r_{W}, r_{t}$, and $r_{\Gamma_{r}}$ are defined as follows;

$$
r_{W}=\frac{m_{W}^{2}}{m_{H_{i}}^{2}}, \quad r_{t}=\frac{m_{t}^{2}}{m_{H_{i}}^{2}}, \quad r_{\Gamma_{t}}=\frac{\Gamma_{t}^{2}}{m_{H_{i}}^{2}}
$$

where $\Gamma_{t}$ is the total decay width of the top quark.

In the case that $m_{H_{i}}>m_{H_{j}}(i \neq j)$, the decay $H_{i}^{ \pm} \rightarrow H_{j}^{ \pm} Z^{*} \rightarrow H_{j}^{ \pm} f \bar{f}$, where $f$ is a SM fermion, is allowed. The decay rate is given by

$$
\Gamma\left(H_{i}^{ \pm} \rightarrow H_{j}^{ \pm} Z^{*} \rightarrow H_{j}^{ \pm} f \bar{f}\right)=\frac{N_{c}^{f} m_{H_{i}}}{192 \pi^{3}}\left(\frac{m_{Z}}{v}\right)^{4} \sin ^{2} 2 \chi\left(\left(C_{V}^{f}\right)^{2}+\left(C_{A}^{f}\right)^{2}\right) \int_{0}^{\left(1-\sqrt{r_{j}}\right)^{2}} \mathrm{~d} x \frac{F\left(x, r_{j}\right)^{3}}{\left(x-r_{Z}\right)^{2}+r_{Z} r_{\Gamma_{Z}}},
$$

where $N_{c}^{f}$ is the color degree of freedom of a fermion $f, r_{Z}$ and $r_{j}$ are defined same with that in Eq. (A7), and $r_{\Gamma_{Z}}$ is the ratio of the squared decay rate of $Z$ boson to squared mass of $H_{i}^{ \pm}$:

$$
r_{\Gamma_{Z}}=\frac{\Gamma_{Z}^{2}}{m_{H_{i}}^{2}}
$$


In addition, the coefficient $C_{V}^{f}\left(C_{A}^{f}\right)$ in Eq. (A10) is the coupling constant of the vector (axial vector) current:

$$
\mathcal{L}=\frac{g_{L}}{2 \cos \theta_{W}} \bar{f} \gamma^{\mu}\left(C_{V}^{f}+C_{A}^{f} \gamma_{5}\right) f Z_{\mu},
$$

where $g_{L}$ is the gauge coupling constant of the gauge group $S U(2)_{L}$, and $\theta_{W}$ is the Weinberg angle. In Eq. (A10), mass of fermions are neglected.

\section{Formulas for decays of the doubly charged scalar $\boldsymbol{\Phi}^{ \pm \pm}$}

\section{a. 2-body decay}

If $m_{\Phi^{ \pm \mp}}>m_{H_{i}}+m_{W}$, the decay $\Phi^{ \pm \pm} \rightarrow H_{i}^{ \pm} W^{ \pm}(i=1$, 2 ) is allowed. The decay rate is given by

$$
\Gamma\left(\Phi^{ \pm \pm} \rightarrow H_{i}^{ \pm} W^{ \pm}\right)=\frac{m_{\Phi}}{8 \pi}\left(\frac{m_{\Phi}}{v}\right)^{2} \chi_{i}^{2} F\left(R_{W}, R_{i}\right)^{3},
$$

where $\chi_{i}$ is defined in Eq. (27), the function $F(x, y)$ is defined in Eq. (A4), and $R_{i}$ and $R_{W}$ is defined as follows;

$$
R_{W}=\frac{m_{W}^{2}}{m_{\Phi}^{2}}, \quad R_{i}=\frac{m_{H_{i}}^{2}}{m_{\Phi}^{2}} .
$$

\section{b. 3-body decay}

In the case that where the mass differences between $\Phi^{ \pm \pm}$ and $H_{i}^{ \pm}$is so small that decays $\Phi^{ \pm \pm} \rightarrow H_{i}^{ \pm} W^{ \pm}$are prohibited, three-body decays $\Phi^{ \pm \pm} \rightarrow H_{i}^{ \pm} f \bar{f}^{\prime}$, where $f$ and $f^{\prime}$ are SM fermions, are dominant in small $m_{\Phi}$ region. (See Fig. 3.) The branching ratio for $\Phi^{ \pm \pm} \rightarrow H_{i}^{ \pm} f \bar{f}^{\prime}$ is given by

$$
\begin{aligned}
& \Gamma\left(\Phi^{ \pm \pm} \rightarrow H_{i}^{ \pm} f \bar{f}^{\prime}\right) \\
& \quad=\frac{N_{c}^{f}}{96 \pi^{3}} \chi_{i}^{2} \int_{0}^{\left(1-\sqrt{R_{i}}\right)^{2}} \frac{\mathrm{d} x}{x} \frac{F\left(x, R_{i}\right)^{3}}{\left(x-R_{W}\right)^{2}+R_{\Gamma_{W}} R_{W}},
\end{aligned}
$$

where $R_{\Gamma_{W}}$ is the squared ratio of the decay width of $W$ boson $\left(\Gamma_{W}\right)$ to $m_{\Phi}$

$$
R_{\Gamma_{W}}=\frac{\Gamma_{W}^{2}}{m_{\Phi}^{2}}
$$

In Eq. (A15), we neglect the masses of $f$ and $f^{\prime}$.

In the large $m_{\Phi}$ region, $\Phi^{ \pm \pm} \rightarrow W^{ \pm} f \bar{f}^{\prime}$ is also important. The decay rate is given by

$$
\begin{aligned}
\Gamma\left(\Phi^{ \pm \pm} \rightarrow W^{ \pm} f \overline{f^{\prime}}\right)= & \frac{N_{c}^{f} m_{\Phi}}{256 \pi^{3}}\left(\frac{m_{\Phi}}{v}\right)^{4} \sin 2 \chi \cot \beta^{2}\left|V_{f f^{\prime}}\right|^{2} \\
& \times \int_{\left(\sqrt{R_{f}}+\sqrt{R_{f^{\prime}}}\right)^{2}}^{\left(1-\sqrt{R_{W}}\right)^{2}} \mathrm{~d} x F\left(\frac{R_{f}}{x}, \frac{R_{f}^{\prime}}{x}\right) \\
& \times F\left(x, R_{W}\right) G(x),
\end{aligned}
$$

where the function $G(x)$ is defined as follows;

$$
\begin{aligned}
G(x)= & \left\{\left(R_{f}+R_{f^{\prime}}\right)\left(x-R_{f}-R_{f^{\prime}}\right)-4 R_{f} R_{f^{\prime}}\right\} \\
& \times\left\{\frac{1}{\left(x-R_{1}\right)^{2}+R_{1} R_{\Gamma_{1}}}+\frac{1}{\left(x-R_{2}\right)^{2}+R_{2} R_{\Gamma_{2}}}\right\}^{2} .
\end{aligned}
$$

The symbols $R_{f}, R_{f^{\prime}}, R_{i}$, and $R_{\Gamma_{i}}(i=1,2)$ are given by $R_{f}=\frac{m_{f}^{2}}{m_{\Phi}^{2}}, \quad R_{f^{\prime}}=\frac{m_{f^{\prime}}^{2}}{m_{\Phi}^{2}}, \quad R_{i}=\frac{m_{H_{i}}^{2}}{m_{\Phi}^{2}}, \quad R_{\Gamma_{i}}=\frac{\Gamma_{H_{i}}^{2}}{m_{\Phi}^{2}}$,

where $m_{f}\left(m_{f^{\prime}}\right)$ is mass of $f\left(f^{\prime}\right)$, and $\Gamma_{H_{i}}$ is the decay width of $H_{i}^{ \pm}$.

\section{APPENDIX B: CONDITIONS FOR THE SCALAR POTENTIAL TO BE BOUNDED FROM BELOW}

In this paper, we suppose that the electric charge is not broken. Even in this case, it is necessary to investigate conditions for the scalar potential to be bounded from below.

Quartic terms in the scalar potential are as follows;

$$
\begin{aligned}
V_{4}= & \frac{\lambda_{1}}{2}\left|\phi_{1}\right|^{4}+\frac{\lambda_{2}}{2}\left|\phi_{2}\right|^{4}+\lambda_{3}\left|\phi_{1}\right|^{2}\left|\phi_{2}\right|^{2}+\lambda_{4}\left|\phi_{1}^{\dagger} \phi_{2}\right|^{2}+\frac{1}{2}\left(\lambda_{5}\left(\phi_{1}^{\dagger} \phi_{2}\right)^{2}+\text { H.c. }\right) \\
& +\frac{1}{2} \lambda_{\Phi}|\Phi|^{4}+\rho_{1}\left|\phi_{1}\right|^{2}|\Phi|^{2}+\rho_{2}\left|\phi_{2}\right|^{2}|\Phi|^{2}+\sigma_{1}\left|\phi_{1}^{\dagger} \Phi\right|^{2}+\sigma_{2}\left|\phi_{2}^{\dagger} \Phi\right|^{2} \\
& +\left\{\kappa\left(\Phi^{\dagger} \phi_{1}\right)\left(\tilde{\phi}_{1}^{\dagger} \phi_{2}\right)+\text { H.c. }\right\} .
\end{aligned}
$$

First, we consider directions where one of the three doublets has a large value. Obviously, conditions for the potential to be bounded from the below in these directions are given by

$$
\lambda_{1}>0, \quad \lambda_{2}>0, \quad \lambda_{\Phi}>0 .
$$


Second, we consider directions where two of the three doublets have large values. Especially, we show details for directions where $\left|\phi_{1}\right|\left(\equiv \sqrt{\left|\phi_{1}\right|^{2}}\right)$ and $\left|\phi_{2}\right|\left(\equiv \sqrt{\left|\phi_{2}\right|^{2}}\right)$ approach to infinity. Conditions for other directions can be derived in the same way. Quartic terms constituted by $\phi_{1}$ and $\phi_{2}$ are as follows;

$$
\begin{aligned}
V_{4}^{(1)}= & \frac{\lambda_{1}}{2}\left|\phi_{1}\right|^{4}+\frac{\lambda_{2}}{2}\left|\phi_{2}\right|^{4}+\lambda_{3}\left|\phi_{1}\right|^{2}\left|\phi_{2}\right|^{2}+\lambda_{4}\left|\phi_{1}^{\dagger} \phi_{2}\right|^{2} \\
& +\frac{\lambda_{5}}{2}\left(\left(\phi_{1}^{\dagger} \phi_{2}\right)^{2}+\text { H.c. }\right)
\end{aligned}
$$

From the Cauchy-Schwarz inequality,

$$
\left|\phi_{1}^{\dagger} \phi_{2}\right| \equiv \sqrt{\left|\phi_{1}^{\dagger} \phi_{2}\right|^{2}} \leq\left|\phi_{1}\right|\left|\phi_{2}\right|,
$$

in the direction where $\phi_{1}$ and $\phi_{2}$ are linearly independent, the terms $\lambda_{4}$ and $\lambda_{5}$ do not need to be considered. The scalar potential is then approximately evaluated as

$V=\frac{r^{4}}{2}\left(\lambda_{1} \cos ^{2} \theta+2 \lambda_{3} \cos \theta \sin \theta+\lambda_{2} \sin ^{2} \theta\right), \quad(r \rightarrow \infty)$,

where we define a polar coordinate as $\left|\phi_{1}\right|^{2}=r^{2} \cos \theta, \quad\left|\phi_{2}\right|^{2}=r^{2} \sin \theta, \quad(0<r, 0<\theta<\pi / 2)$.

The condition for the potential to be bounded from below includes

$$
\lambda_{1} \cos ^{2} \theta+2 \lambda_{3} \cos \theta \sin \theta+\lambda_{2} \sin ^{2} \theta>0, \quad(0<\theta<\pi / 2) .
$$

This inequality yields

$$
\lambda_{1}>0, \quad \lambda_{2}>0, \quad \lambda_{3}>-\sqrt{\lambda_{1} \lambda_{2}} .
$$

Next, we consider the direction where $\phi_{1}$ is proportional to $\phi_{2}$, and $\left|\phi_{1}\right|$ and $\left|\phi_{2}\right|$ approach to infinity. We then have to consider the terms $\lambda_{4}$ and $\lambda_{5}$. In the same way of deriving the condition in Eq. (B8), conditions for the potential to be bounded from below in this direction is given by as follows.

$$
\lambda_{1}>0, \quad \lambda_{2}>0, \quad \lambda_{3}+\lambda_{4}-\left|\lambda_{5}\right|>-\sqrt{\lambda_{1} \lambda_{2}} .
$$

The conditions in Eqs. (B8) and (B9) are same with those in the THDM [14,70,71].

For directions where other two doublets have large values, the following conditions can be derived in the same way.

$$
\begin{array}{lll}
\lambda_{1}>0, \lambda_{2}>0, & \lambda_{\Phi}>0, \quad \rho_{1}>-\sqrt{\lambda_{1} \lambda_{\Phi}}, & \rho_{1}+\sigma_{1}>-\sqrt{\lambda_{1} \lambda_{\Phi}}, \\
\rho_{2}>-\sqrt{\lambda_{2} \lambda_{\Phi}}, & \rho_{2}+\sigma_{2}>-\sqrt{\lambda_{2} \lambda_{\Phi}} .
\end{array}
$$

Third, we consider directions where all of three doublets have large values. In directions where $\phi_{1}, \phi_{2}$, and $\Phi$ are linearly independent, the scalar potential is approximately evaluated as

$$
V=\frac{\lambda_{1}}{2}\left|\phi_{1}\right|^{4}+\frac{\lambda_{2}}{2}\left|\phi_{2}\right|^{4}+\lambda_{3}\left|\phi_{1}\right|^{2}\left|\phi_{2}\right|^{2}+\frac{1}{2} \lambda_{\Phi}|\Phi|^{4}+\rho_{1}\left|\phi_{1}\right|^{2}|\Phi|^{2}+\rho_{2}\left|\phi_{2}\right|^{2}|\Phi|^{2} .
$$

We define a polar coordinate as

$$
\begin{aligned}
\left|\phi_{1}\right|^{2} & =r^{2} \cos \theta, \quad\left|\phi_{2}\right|^{2}=r^{2} \sin \theta \cos \varphi, \\
|\Phi|^{2} & =r^{2} \sin \theta \sin \varphi, \quad(0<r, 0<\theta<\pi / 2,0<\phi<\pi / 2) .
\end{aligned}
$$

The condition for the potential to be bounded from below includes

$$
\lambda_{1} \cos ^{2} \theta+2 \sin \theta \cos \theta\left(\lambda_{3} \cos \varphi+\rho_{1} \sin \varphi\right)+\left(\lambda_{2} \cos ^{2} \varphi+2 \rho_{2} \sin \varphi \cos \varphi+\lambda_{\Phi} \sin ^{2} \varphi\right) \sin ^{2} \theta>0 .
$$

In the same way to derive Eq. (B8), we obtain

$$
\lambda_{1}>0, \quad \lambda_{2} \cos ^{2} \varphi+2 \rho_{2} \sin \varphi \cos \varphi+\lambda_{\Phi} \sin ^{2} \varphi>0, \quad F_{1}(\varphi)>0, \quad(0<\varphi<\pi / 2),
$$

where

$$
F_{1}(\varphi)=\lambda_{3} \cos \varphi+\rho_{1} \sin \varphi+\sqrt{\lambda_{1}\left(\lambda_{2} \cos ^{2} \varphi+2 \rho_{2} \sin \varphi \cos \varphi+\lambda_{\Phi} \sin ^{2} \varphi\right)} .
$$


From the second in equality, we obtain $\lambda_{2}>0, \lambda_{\Phi}>0$ and $\rho_{2}>-\sqrt{\lambda_{2} \lambda_{\Phi}}$, and they are already included in Eq. (B10). Therefore, the independent condition is only the third inequality in Eq. (B14).

Next, we consider conditions some especial directions where $\phi_{1} \propto \phi_{2}, \phi_{1} \propto \Phi, \phi_{2} \propto \Phi$, and $\phi_{1} \propto \phi_{2} \propto \Phi$. In the same way of deriving Eq. (B14), conditions for these directions are given by

$$
F_{1}(\varphi)+\left(\lambda_{4}-\left|\lambda_{5}\right|\right) \cos \varphi>0
$$

$$
\begin{gathered}
F_{1}(\varphi)+\sigma_{1} \sin \varphi>0, \\
F_{2}(\varphi)>0, \\
F_{2}(\varphi)+\left(\lambda_{4}-\left|\lambda_{5}\right|\right) \cos \varphi+\sigma_{1} \sin \varphi>0,
\end{gathered}
$$

for $0<\varphi<\pi / 2$, where

$$
F_{2}(\varphi)=\lambda_{3} \cos \varphi+\rho_{1} \sin \varphi+\sqrt{\lambda_{1}\left(\lambda_{2} \cos ^{2} \varphi+2\left(\rho_{2}+\sigma_{2}\right) \sin \varphi \cos \varphi+\lambda_{\Phi} \sin ^{2} \varphi\right)}
$$

Finally, we consider the direction where $\phi_{1} \propto \Phi \propto \tilde{\phi}_{2}$. The term $\kappa\left(\Phi^{\dagger} \phi_{1}\right)\left(\tilde{\phi}_{1}^{\dagger} \phi_{2}\right)$ then approaches to infinity when $\left|\phi_{1}\right| \rightarrow \infty,\left|\phi_{2}\right| \rightarrow \infty$, and $|\Phi| \rightarrow \infty$. The condition for the potential to be bounded from below in this direction is given by

$$
F_{1}(\varphi)+\sigma_{1} \sin \varphi-2|\kappa| \sqrt{\sin \varphi \cos \varphi}>0, \quad(0<\varphi<\pi / 2) .
$$

Finally, we have derived all conditions for the potential to be bounded from below. They are summarized in the following:

$$
\begin{aligned}
& \lambda_{1}>0, \lambda_{2}>0, \quad \lambda_{\Phi}>0, \quad \lambda_{3}>-\sqrt{\lambda_{1} \lambda_{2}}, \quad \lambda_{3}+\lambda_{4}-\left|\lambda_{5}\right|>-\sqrt{\lambda_{1} \lambda_{2}}, \\
& \rho_{1}>-\sqrt{\lambda_{1} \lambda_{\Phi}}, \quad \rho_{1}+\sigma_{1}>-\sqrt{\lambda_{1} \lambda_{\Phi}}, \quad \rho_{2}>-\sqrt{\lambda_{2} \lambda_{\Phi}}, \quad \rho_{2}+\sigma_{2}>-\sqrt{\lambda_{2} \lambda_{\Phi}}, \\
& F_{1}(\varphi)>0, \quad F_{1}(\varphi)+\left(\lambda_{4}-\left|\lambda_{5}\right|\right) \cos \varphi>0, \quad F_{1}(\varphi)+\sigma_{1} \sin \varphi>0, \\
& F_{2}(\varphi)>0, \quad F_{2}(\varphi)+\left(\lambda_{4}-\left|\lambda_{5}\right|\right) \cos \varphi+\sigma_{1} \sin \varphi>0, \\
& F_{1}(\varphi)+\sigma_{1} \sin \varphi-2|\kappa| \sqrt{\sin \varphi \cos \varphi}>0, \quad \text { for } \varphi \in(0, \pi / 2) .
\end{aligned}
$$

\section{Scenario I}

In this subsection, we investigate the conditions for the potential to be bounded from below in Scenario I. In Scenario I, we assume that

$$
\begin{aligned}
& m_{\Phi}=200 \mathrm{GeV}, \quad m_{H_{1}}=100 \mathrm{GeV}, \quad m_{H_{2}}=120 \mathrm{GeV}, \quad \tan \beta=10, \quad \chi=\frac{\pi}{4}, \\
& m_{H}=130 \mathrm{GeV}, \quad m_{A}=140 \mathrm{GeV} .
\end{aligned}
$$

These input parameters can be realized by, for example, the following scalar coupling constants.

$$
\begin{aligned}
& \mu_{3}=38.6 \mathrm{GeV}, \quad \mu_{\Phi}=191 \mathrm{GeV}, \quad \lambda_{1}=3.30, \quad \lambda_{2}=0.259, \quad \lambda_{3}=-0.786, \quad \lambda_{4}=1.09, \\
& \lambda_{5}=-0.075, \quad \kappa=-0.731, \quad \rho_{1}=1.5, \quad \rho_{2}=0.1 .
\end{aligned}
$$

In addition, we assume the following coupling constants ${ }^{10}$;

$$
\sigma_{1}=2.0, \quad \sigma_{2}=0.1, \quad \lambda_{\Phi}=1.5
$$

In this case, the conditions

$$
\begin{aligned}
& \lambda_{1}>0, \quad \lambda_{2}>0, \quad \lambda_{\Phi}>0, \quad \lambda_{3}>-\sqrt{\lambda_{1} \lambda_{2}}, \quad \lambda_{3}+\lambda_{4}-\left|\lambda_{5}\right|>-\sqrt{\lambda_{1} \lambda_{2}}, \\
& \rho_{1}>-\sqrt{\lambda_{1} \lambda_{\Phi}}, \quad \rho_{1}+\sigma_{1}>-\sqrt{\lambda_{1} \lambda_{\Phi}}, \quad \rho_{2}>-\sqrt{\lambda_{2} \lambda_{\Phi}}, \quad \rho_{2}+\sigma_{2}>-\sqrt{\lambda_{2} \lambda_{\Phi}},
\end{aligned}
$$

\footnotetext{
${ }^{10}$ These coupling constants are irrelevant to the mass spectrums of the scalars. However, they contribute to cubic couplings between the Higgs boson and the charged scalars. See Appendix D.
} 
are obviously satisfied. Left conditions include functions of $\varphi$ in the left-hand sides of inequalities. In Fig. 13, we show these functions, and all of them have to be positive in order to bound the potential from below. The minimum value of $F_{1}(\varphi)+\sigma_{1} \sin \varphi-2|\kappa| \sqrt{\sin \varphi \cos \varphi}$ (the blue dotted line) is closed to 0 , however it is about 0.033 . Therefore, all conditions are satisfied, and the scalar potential in Scenario I is bounded from below.

\section{Scenario II}

In this subsection, we investigate the conditions for the potential to be bounded from below in Scenario II. In Scenario II, we assume that

$$
\begin{aligned}
& m_{\Phi}=300 \mathrm{GeV}, \quad m_{H_{1}}=200 \mathrm{GeV}, \quad m_{H_{2}}=250 \mathrm{GeV}, \quad \tan \beta=3, \quad \chi=\frac{\pi}{4}, \\
& m_{H}=210 \mathrm{GeV}, \quad m_{A}=220 \mathrm{GeV} .
\end{aligned}
$$

These input parameters can be realized by, for example, the following scalar coupling constants.

$$
\begin{aligned}
& \mu_{3}=95.3 \mathrm{GeV}, \quad \mu_{\Phi}=247 \mathrm{GeV}, \quad \lambda_{1}=2.32, \quad \lambda_{2}=0.284, \quad \lambda_{3}=-0.557, \quad \lambda_{4}=0.886, \\
& \lambda_{5}=-0.30, \quad \kappa=-1.18, \quad \rho_{1}=1.5, \quad \rho_{2}=0.90 .
\end{aligned}
$$

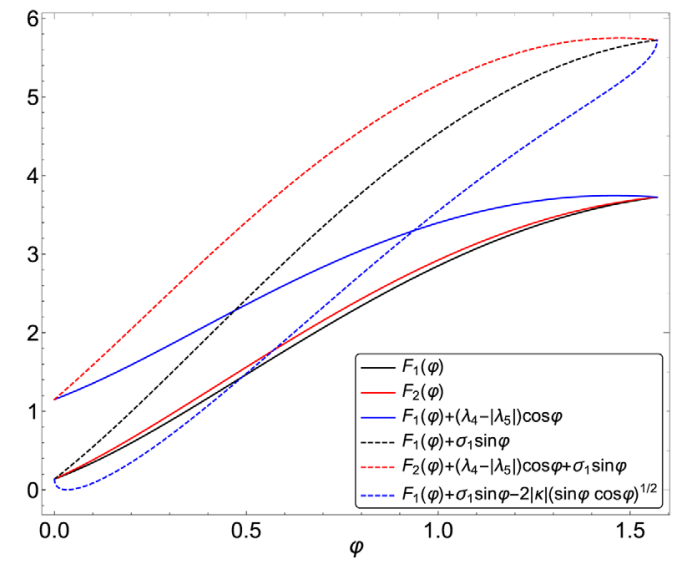

FIG. 13. Conditions for the potential to be bounded from below in Scenario I.

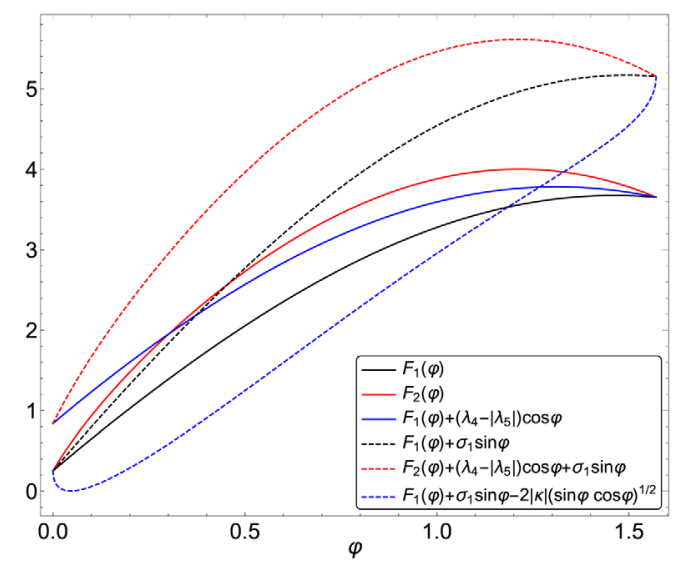

FIG. 14. Conditions for the potential to be bounded from below in Scenario II.
In addition, we assume the following coupling constants;

$$
\sigma_{1}=1.5, \quad \sigma_{2}=1.5, \quad \lambda_{\Phi}=2.0 .
$$

Then, as in the case of Scenario I, conditions in Eq. (B26) are obviously satisfied. In Fig. 14, left-hand sides of inequalities which are not included in Eq. (B26) are shown. The minimum value of $F_{1}(\varphi)+\sigma_{1} \sin \varphi-2|\kappa| \sqrt{\sin \varphi \cos \varphi}$ (the blue dotted line) is closed to 0 , however it is about 0.046 . Therefore, all conditions are satisfied, and the scalar potential in Scenario II is bounded from below.

\section{APPENDIX C: THE OBLIQUE PARAMETERS}

In this section, we discuss constraints on masses of new scalars from oblique parameters $S, T$, and $U$ [49]. By fixing $U=0, S$ and $T$ are constrained as follows $[62]^{11}$;

$$
S=0.00 \pm 0.07, \quad T=0.05 \pm 0.06 .
$$

Here, we consider only the $T$ parameter. In the model, the $T$ parameter from scalar loop diagrams is given by

$$
\begin{aligned}
T= & \frac{\sqrt{2} G_{F}}{16 \pi^{2} \alpha}\left[c_{\beta-\alpha}^{2} c_{\chi}^{2} \Delta F\left(m_{H_{1}}, m_{h}\right)+c_{\beta-\alpha}^{2} s_{\chi}^{2} \Delta F\left(m_{H_{2}}, m_{h}\right)\right. \\
& +s_{\beta-\alpha}^{2} c_{\chi}^{2} \Delta F\left(m_{H_{1}}, m_{H}\right)+s_{\beta-\alpha}^{2} s_{\chi}^{2} \Delta F\left(m_{H_{2}}, m_{H}\right) \\
& +c_{\chi}^{2} \Delta F\left(m_{H_{1}}, m_{A}\right)+s_{\chi}^{2} \Delta F\left(m_{H_{2}}, m_{A}\right) \\
& +2 s_{\chi}^{2} \Delta F\left(m_{\Phi}, m_{H_{1}}\right)+2 c_{\chi}^{2} \Delta F\left(m_{\Phi}, m_{H_{2}}\right) \\
& -c_{\beta-\alpha}^{2} \Delta F\left(m_{A}, m_{h}\right)-s_{\beta-\alpha}^{2} \Delta F\left(m_{A}, m_{H}\right) \\
& \left.-2 s_{2 \chi}^{2} \Delta F\left(m_{H_{1}}, m_{H_{2}}\right)\right],
\end{aligned}
$$

\footnotetext{
${ }^{11}$ By definition of $S, T$, and $U$, the $\mathrm{SM}$ prediction is $S=T=0$.
} 
where $c_{\theta}=\cos \theta, s_{\theta}=\sin \theta$, and the function $\Delta F\left(m_{1}, m_{2}\right)$ is defined as follows;

$$
\Delta F\left(m_{1}, m_{2}\right)=\frac{m_{1}^{2}+m_{2}^{2}}{2}-\frac{m_{1}^{2} m_{2}^{2}}{m_{2}^{2}-m_{1}^{2}} \log \left(\frac{m_{2}^{2}}{m_{1}^{2}}\right) .
$$

In the case that $\sin (\beta-\alpha)=1$ (the alignment limit) and $\chi=\pi / 4, T$ can be simplified as

$$
\begin{aligned}
T= & \frac{\sqrt{2} G_{F}}{16 \pi^{2} \alpha}\left[\frac{1}{2} \Delta F\left(m_{H_{1}}, m_{H}\right)+\frac{1}{2} \Delta F\left(m_{H_{2}}, m_{H}\right)+\frac{1}{2} \Delta F\left(m_{H_{1}}, m_{A}\right)+\frac{1}{2} \Delta F\left(m_{H_{2}}, m_{A}\right)\right. \\
& \left.+\Delta F\left(m_{\Phi}, m_{H_{1}}\right)+\Delta F\left(m_{\Phi}, m_{H_{2}}\right)-\Delta F\left(m_{A}, m_{H}\right)-2 \Delta F\left(m_{H_{1}}, m_{H_{2}}\right)\right] .
\end{aligned}
$$

By using the mass spectra in Scenario I and II, $T$ in the scenarios are predicted as

$$
T \simeq \begin{cases}0.0099 & (\text { Scenario I }) \\ 0.0052 & (\text { Scenario II })\end{cases}
$$

By comparing Eqs. (C1) and (C5), it is concluded that in bosh scenarios, the $T$ parameter are consistent with the current constraint.

\section{APPENDIX D: THE DIPHOTON DECAY OF THE HIGGS BOSON}

In this section, we discuss constraints from the measurement of $h \rightarrow \gamma \gamma$ at the LHC. The current limit is given by

$$
\left(\sigma \times B_{\gamma \gamma}\right)_{\mathrm{obs}}=127 \pm 10 \mathrm{fb}
$$

where $\sigma$ is the cross section of the Higgs production, and $B_{\gamma \gamma}$ is the branching ratio of the diphoton decay of the Higgs boson [63]. On the other hand, the expectation value in the SM is as follows [63];

$$
\left(\sigma \times B_{\gamma \gamma}\right)_{\mathrm{SM}}=116 \pm 5 \mathrm{fb} .
$$

In this model, the diphoton decay is generated by oneloop diagrams of charged scalars in addition to those of the SM charged particles. The decay rate is given by

$$
\begin{aligned}
\Gamma_{\gamma \gamma}= & \frac{G_{F} \alpha^{2} m_{h}^{3}}{128 \sqrt{2} \pi^{3}} \mid A_{W}\left(\frac{m_{h}^{2}}{4 m_{W}^{2}}\right)+\sum_{f} Q_{f}^{2} N_{c}^{f} A_{F}\left(\frac{m_{h}^{2}}{4 m_{f}^{2}}\right) \\
& +\left.\frac{v^{2}}{2 m_{h}^{2}} \sum_{s=H_{1}, H_{2}, \Phi} \frac{\xi_{s}}{v} Q_{s}^{2} A_{S}\left(\frac{m_{h}^{2}}{4 m_{s}^{2}}\right)\right|^{2},
\end{aligned}
$$

where $f$ is the SM fermion, and $Q_{f}\left(N_{c}^{f}\right)$ is the electric charge (the color degree of freedom) of $f[72,73]$. The parameter $m_{W}, m_{f}$, and $m_{s}$ are masses of $\mathrm{W}$ boson, a fermion $f$, and a charged scalar $s^{ \pm( \pm)}$, respectively. The functions $A_{W}(r), A_{F}(r), A_{S}(r)$ are defined as follows;

$$
A_{W}(r)=-\frac{1}{r^{2}}\left(2 r^{2}+3 r+3(2 r-1) f(r)\right),
$$

$$
\begin{gathered}
A_{F}(r)=\frac{2}{r^{2}}(r+(r-1) f(r)), \\
A_{S}(r)=-\frac{1}{r^{2}}(r-f(r)),
\end{gathered}
$$

where

$$
f(r)= \begin{cases}(\operatorname{Arcsin} \sqrt{r})^{2} & r<1 \\ -\frac{1}{4}\left[\log \frac{1+\sqrt{1-r^{-1}}}{1-\sqrt{1-r^{-1}}}-i \pi\right] & r>1\end{cases}
$$

In addition, $Q_{s}$ is the electric charge of a charged scalar $s$, and $\xi_{s}$ is cubic coupling constant between the Higgs boson and a charged scalar $s^{ \pm( \pm)}\left(s=H_{1}, H_{2}, \Phi\right)$;

$$
\mathcal{L}_{h s s}=\xi_{H_{1}} h\left|H_{1}^{+}\right|^{2}+\xi_{H_{2}} h\left|H_{2}^{+}\right|^{2}+\xi_{\Phi} h\left|\Phi^{++}\right|^{2} .
$$

By using parameters in the scalar potential, the coupling constants $\xi_{s}\left(s=H_{1}, H_{2}, \Phi\right)$ are given by

$$
\begin{aligned}
& \xi_{H_{1}}= v\left(\lambda_{1} c_{\beta}^{2} s_{\beta}^{2} c_{\chi}^{2}+\lambda_{2} s_{\beta}^{2} c_{\beta}^{2} c_{\chi}^{2}+\lambda_{3} s_{\beta}^{4} c_{\chi}^{2}+\lambda_{3} c_{\beta}^{4} c_{\chi}^{2}\right. \\
&-2 \lambda_{4} s_{\beta}^{2} c_{\beta}^{2} c_{\chi}^{2}-2 \lambda_{5} s_{\beta}^{2} c_{\beta}^{2} c_{\chi}^{2}+\left(\rho_{1}+\sigma_{1}\right) c_{\beta}^{2} s_{\chi}^{2} \\
&\left.+\left(\rho_{2}+\sigma_{2}\right) s_{\beta}^{2} s_{\chi}^{2}+2 \kappa c_{\beta} s_{\chi} c_{\chi}\right), \\
& \xi_{H_{2}}= v\left(\lambda_{1} c_{\beta}^{2} s_{\beta}^{2} s_{\chi}^{2}+\lambda_{2} s_{\beta}^{2} c_{\beta}^{2} s_{\chi}^{2}+\lambda_{3} s_{\beta}^{4} s_{\chi}^{2}+\lambda_{3} c_{\beta}^{4} s_{\chi}^{2}\right. \\
&-2 \lambda_{4} s_{\beta}^{2} c_{\beta}^{2} s_{\chi}^{2}-2 \lambda_{5} s_{\beta}^{2} c_{\beta}^{2} s_{\chi}^{2}+\left(\rho_{1}+\sigma_{1}\right) c_{\beta}^{2} s_{\chi}^{2} \\
&\left.+\left(\rho_{2}+\sigma_{2}\right) s_{\beta}^{2} c_{\chi}^{2}-2 \kappa c_{\beta} s_{\chi} c_{\chi}\right), \\
& \xi_{\Phi}=v\left(\rho_{1} c_{\beta}^{2}+\rho_{2} s_{\beta}^{2}\right) .
\end{aligned}
$$

We assume that new physics does not contribute to the Higgs production processes. The value of $\left(\sigma \times B_{\gamma \gamma}\right)$ in the model is then given by

$$
\left(\sigma \times B_{\gamma \gamma}\right)_{Y=3 / 2}=\left(\sigma \times B_{\gamma \gamma}\right)_{\mathrm{SM}} \times \frac{\left(B_{\gamma \gamma}\right)_{Y=3 / 2}}{\left(B_{\gamma \gamma}\right)_{\mathrm{SM}}} .
$$


By using the benchmark values of scalar coupling constants in Subsections B 1 and B 2 and Eqs. (D3) and (D12), $\sigma \times$ $B_{\gamma \gamma}$ in the scenarios are predicted as

$$
\left(\sigma \times B_{\gamma \gamma}\right)_{Y=3 / 2} \simeq \begin{cases}132 \mathrm{fb} & (\text { Scenario I }) \\ 120 \mathrm{fb} & (\text { Scenario II }) .\end{cases}
$$

By comparing Eqs. (D1) and (D13), it is concluded that these predictions are consistent with the current measurement at the LHC. On the other hand, this deviation from the SM prediction might be detected at future high-energy colliders, for example the HL-LHC or the international linear collider (ILC).
[1] G. Aad et al. (ATLAS Collaboration), Phys. Lett. B 716, 1 (2012); S. Chatrchyan et al. (CMS Collaboration), Phys. Lett. B 716, 30 (2012).

[2] P. Minkowski, Phys. Lett. 67B, 421 (1977); T. Yanagida, Conf. Proc. C 7902131, 95 (1979); KEK-79-18-95; Prog. Theor. Phys. 64, 1103 (1980); M. Gell-Mann, P. Ramond, and R. Slansky, Conf. Proc. C 790927, 315 (1979); R. N. Mohapatra and G. Senjanovic, Phys. Rev. Lett. 44, 912 (1980).

[3] W. Konetschny and W. Kummer, Phys. Lett. 70B, 433 (1977); M. Magg and C. Wetterich, Phys. Lett. 94B, 61 (1980); J. Schechter and J. W. F. Valle, Phys. Rev. D 22, 2227 (1980); G. Lazarides, Q. Shafi, and C. Wetterich, Nucl. Phys. B181, 287 (1981).

[4] R. N. Mohapatra and G. Senjanovic, Phys. Rev. Lett. 44, 912 (1980); Phys. Rev. D 23, 165 (1981).

[5] R. Foot, H. Lew, X. G. He, and G. C. Joshi, Z. Phys. C 44, 441 (1989).

[6] A. Zee, Phys. Lett. 93B, 389 (1980); 95B, 461(E) (1980).

[7] A. Zee, Nucl. Phys. B264, 99 (1986); K. S. Babu, Phys. Lett. B 203, 132 (1988).

[8] T. P. Cheng and L. F. Li, Phys. Rev. D 22, 2860 (1980).

[9] L. M. Krauss, S. Nasri, and M. Trodden, Phys. Rev. D 67, 085002 (2003).

[10] E. Ma, Phys. Rev. D 73, 077301 (2006).

[11] M. Aoki, S. Kanemura, and O. Seto, Phys. Rev. Lett. 102, 051805 (2009); Phys. Rev. D 80, 033007 (2009); M. Aoki, S. Kanemura, and K. Yagyu, Phys. Rev. D 83, 075016 (2011).

[12] M. Gustafsson, J. M. No, and M. A. Rivera, Phys. Rev. Lett. 110, 211802 (2013); 112, 259902(E) (2014); Phys. Rev. D 90, 013012 (2014).

[13] T. Araki, C. Q. Geng, and K. I. Nagao, Phys. Rev. D 83, 075014 (2011).

[14] N. G. Deshpande and E. Ma, Phys. Rev. D 18, 2574 (1978).

[15] J. McDonald, Phys. Rev. D 50, 3637 (1994); C. P. Burgess, M. Pospelov, and T. ter Veldhuis, Nucl. Phys. B619, 709 (2001); S. Kanemura, S. Matsumoto, T. Nabeshima, and N. Okada, Phys. Rev. D 82, 055026 (2010).

[16] M. Kobayashi and T. Maskawa, Prog. Theor. Phys. 49, 652 (1973).

[17] T. D. Lee, Phys. Rev. D 8, 1226 (1973).

[18] V. A. Kuzmin, V. A. Rubakov, and M. E. Shaposhnikov, Phys. Lett. 155B, 36 (1985).

[19] A. G. Cohen, D. B. Kaplan, and A. E. Nelson, Nucl. Phys. B349, 727 (1991).
[20] M. Aoki, S. Kanemura, and K. Yagyu, Phys. Lett. B 702, 355 (2011); 706, 495(E) (2012).

[21] H. Okada and K. Yagyu, Phys. Rev. D 93, 013004 (2016).

[22] K. Cheung and H. Okada, Phys. Lett. B 774, 446 (2017).

[23] K. Enomoto, S. Kanemura, K. Sakurai, and H. Sugiyama, Phys. Rev. D 100, 015044 (2019).

[24] E. Ma, Phys. Lett. B 809, 135736 (2020).

[25] A. Das, K. Enomoto, S. Kanemura, and K. Yagyu, Phys. Rev. D 101, 095007 (2020).

[26] H. Georgi and M. Machacek, Nucl. Phys. B262, 463 (1985).

[27] N. Arkani-Hamed, A. G. Cohen, E. Katz, and A. E. Nelson, J. High Energy Phys. 07 (2002) 034.

[28] J. F. Gunion, Int. J. Mod. Phys. A 11, 1551 (1996).

[29] A. G. Akeroyd and M. Aoki, Phys. Rev. D 72, 035011 (2005); A. G. Akeroyd, C. W. Chiang, and N. Gaur, J. High Energy Phys. 11 (2010) 005; A. G. Akeroyd and H. Sugiyama, Phys. Rev. D 84, 035010 (2011); M. Aoki, S. Kanemura, and K. Yagyu, Phys. Rev. D 85, 055007 (2012).

[30] T. Han, B. Mukhopadhyaya, Z. Si, and K. Wang, Phys. Rev. D 76, 075013 (2007).

[31] S. Kanemura, M. Kikuchi, K. Yagyu, and H. Yokoya, Phys. Rev. D 90, 115018 (2014); Prog. Theor. Exp. Phys. 2015, 51B02 (2015).

[32] V. Rentala, W. Shepherd, and S. Su, Phys. Rev. D 84, 035004 (2011).

[33] S. F. King, A. Merle, and L. Panizzi, J. High Energy Phys. 11 (2014) 124.

[34] H. Sugiyama, K. Tsumura, and H. Yokoya, Phys. Lett. B 717, 229 (2012); A. Alloul, M. Frank, B. Fuks, and M. Rausch de Traubenberg, Phys. Rev. D 88, 075004 (2013); T. Nomura, H. Okada, and H. Yokoya, Nucl. Phys. B929, 193 (2018).

[35] R. Vega and D. A. Dicus, Nucl. Phys. B329, 533 (1990).

[36] T. Han, H. E. Logan, B. McElrath, and L. T. Wang, Phys. Rev. D 67, 095004 (2003).

[37] S. Kanemura, M. Kikuchi, and K. Yagyu, Phys. Rev. D 88, 015020 (2013); J. Hisano and K. Tsumura, Phys. Rev. D 87, 053004 (2013).

[38] ATLAS Collaboration, Technical design report: A highgranularity timing detector for the ATLAS phase-II upgrade, ATLAS-TDR-031, 2020; CMS Collaboration, The phase-2 upgrade of the CMS level-1 trigger, CMS-TDR-021, 2020.

[39] S. L. Glashow and S. Weinberg, Phys. Rev. D 15, 1958 (1977).

[40] M. Aoki, S. Kanemura, K. Tsumura, and K. Yagyu, Phys. Rev. D 80, 015017 (2009). 
[41] N. Cabibbo, Phys. Rev. Lett. 10, 531 (1963).

[42] T. Enomoto and R. Watanabe, J. High Energy Phys. 05 (2016) 002.

[43] J. Haller, A. Hoecker, R. Kogler, K. Mönig, T. Peiffer, and J. Stelzer, Eur. Phys. J. C 78, 675 (2018).

[44] A. Arbey, F. Mahmoudi, O. Stal, and T. Stefaniak, Eur. Phys. J. C 78, 182 (2018).

[45] M. Aiko, S. Kanemura, M. Kikuchi, K. Mawatari, K. Sakurai, and K. Yagyu, Nucl. Phys. B966, 115375 (2021).

[46] G. Abbiendi et al. (ALEPH, DELPHI, L3, OPAL, and LEP Collaborations), Eur. Phys. J. C 73, 2463 (2013).

[47] A. M. Sirunyan et al. (CMS Collaboration), J. High Energy Phys. 07 (2019) 142.

[48] G. Aad et al. (ATLAS Collaboration), J. High Energy Phys. 06 (2021) 145.

[49] M. E. Peskin and T. Takeuchi, Phys. Rev. Lett. 65, 964 (1990); M. E. Peskin and T. Takeuchi, Phys. Rev. D 46, 381 (1992).

[50] M. Capdequi Peyranere, H. E. Haber, and P. Irulegui, Phys. Rev. D 44, 191 (1991); S. Kanemura, Phys. Rev. D 61, 095001 (2000).

[51] E. Ma, D. P. Roy, and J. Wudka, Phys. Rev. Lett. 80, 1162 (1998).

[52] J. F. Gunion and H. E. Haber, Nucl. Phys. B278, 449 (1986); B402, 567(E) (1993); S. S. D. Willenbrock, Phys. Rev. D 35, 173 (1987); O. Brein and W. Hollik, Eur. Phys. J. C 13, 175 (2000); A. A. Barrientos Bendezu and B. A. Kniehl, Phys. Rev. D 64, 035006 (2001).

[53] S. Kanemura and C. P. Yuan, Phys. Lett. B 530, 188 (2002); Q. H. Cao, S. Kanemura, and C. P. Yuan, Phys. Rev. D 69 , 075008 (2004); A. Belyaev, Q. H. Cao, D. Nomura, K. Tobe, and C. P. Yuan, Phys. Rev. Lett. 100, 061801 (2008).

[54] J. F. Gunion, H. E. Haber, F. E. Paige, W. K. Tung, and S. S. D. Willenbrock, Nucl. Phys. B294, 621 (1987).

[55] S. Moretti and K. Odagiri, Phys. Rev. D 55, 5627 (1997).

[56] D. A. Dicus, J. L. Hewett, C. Kao, and T. G. Rizzo, Phys. Rev. D 40, 787 (1989); A. A. Barrientos Bendezu and B. A. Kniehl, Phys. Rev. D 59, 015009 (1998); S. Moretti and K. Odagiri, Phys. Rev. D 59, 055008 (1999).
[57] E. Asakawa, O. Brein, and S. Kanemura, Phys. Rev. D 72, 055017 (2005).

[58] A. A. Barrientos Bendezu and B. A. Kniehl, Phys. Rev. D 61, 097701 (2000); O. Brein, W. Hollik, and S. Kanemura, Phys. Rev. D 63, 095001 (2001).

[59] A. G. Akeroyd, M. Aoki, A. Arhrib, L. Basso, I. F. Ginzburg, R. Guedes, J. Hernandez-Sanchez, K. Huitu, T. Hurth, M. Kadastik et al., Eur. Phys. J. C 77, 276 (2017).

[60] J. Alwall, R. Frederix, S. Frixione, V. Hirschi, F. Maltoni, O. Mattelaer, H. S. Shao, T. Stelzer, P. Torrielli, and M. Zaro, J. High Energy Phys. 07 (2014) 079.

[61] N. D. Christensen and C. Duhr, Comput. Phys. Commun. 180, 1614 (2009); A. Alloul, N. D. Christensen, C. Degrande, C. Duhr, and B. Fuks, Comput. Phys. Commun. 2014) 2250, ,185).

[62] P. A. Zyla et al. (Particle Data Group), Prog. Theor. Exp. Phys. 2020, 083 C01 (2020).

[63] ATLAS Collaboration, ATLAS-CONF-2020-026 (2020), http://cds.cern.ch/record/2725727.

[64] A. M. Sirunyan et al. (CMS Collaboration), J. Instrum. 13, P10005 (2018).

[65] K. Hagiwara, T. Li, K. Mawatari, and J. Nakamura, Eur. Phys. J. C 73, 2489 (2013).

[66] A. Ballestrero, B. Biedermann, S. Brass, A. Denner, S. Dittmaier, R. Frederix, P. Govoni, M. Grossi, B. Jäger, A. Karlberg et al., Eur. Phys. J. C 78, 671 (2018).

[67] G. Aad et al. (ATLAS Collaboration), arXiv:2007.02645.

[68] S. Chatrchyan et al. (CMS Collaboration), J. Instrum. 5, T03022 (2010).

[69] A. M. Sirunyan et al. (CMS Collaboration), J. Instrum. 13, P05011 (2018).

[70] S. Nie and M. Sher, Phys. Lett. B 449, 89 (1999).

[71] S. Kanemura, T. Kasai, and Y. Okada, Phys. Lett. B 471, 182 (1999).

[72] J. R. Ellis, M. K. Gaillard, and D. V. Nanopoulos, Nucl. Phys. B106, 292 (1976).

[73] M. A. Shifman, A. I. Vainshtein, M. B. Voloshin, and V. I. Zakharov, Sov. J. Nucl. Phys. 30, 711 (1979). 